\title{
Zur Klinik und Systematik der assoziativen Aphasie.
}

\author{
Von \\ Dr. Theodor Hoepfner (früher Eisenach)
}

(Z. Z. prakt. Arzt in Saalf $\in$ ld, Ostpr.)

(Eingegangen am 21. April 1922.)

I.

Die populäre Bezeichnung für ein gestörtes Sprechen, dessen Eigentümlichkeit in einer Wiederholung von Silben und Buchstaben an den Anfängen von Silben und Worten besteht, ist "Stottern". Wissenschaftlich muß die Verwendung dieses Ausdrucks durchaus beschränkt werden auf diejenigen Fälle, in denen die primäre Erscheinungsform des Zufälligen, Unwillkürlichen vorliegt. Hierfür paßt besser der aus der Bewegungsphysiologie entnommene Ausdruck „Ataxie“, weil die Eigenart der Erscheinungen sowohl der beginnenden wie der ausgebildeten Störung in das Gebiet der zentralen Regulierung der Willkürbewegungen weist. Die Störungserscheinungen liegen innerhalb der physiologischen Möglichkeiten.

Zur näheren Begründung verweise ich auf das Kapitel 2 „Psychophysiologische Untersuchung" meiner Arbeit „Stottern als assoziative Aphasie" (Zeitschr. f. Pathopsychologie, 1912, Heft 2/3, S. 448 bis 553) hinsichtlich der theoretischen Untersuchungen über Ataxie und resümiere wie dort:

1. „Ataktische Bewegungen sind solche, deren zentrale Regulierung noch nicht von einem geordneten, d. h. die notwendigen Erfahrungsoder Vorstellungselemente enthaltenden Willen vorgenommen wird: primär ataktische Bewegungen (z. B. Sprechenlernen kleiner Kinder, Stolpern usw.)

2. Ataktische Bewegungen sind auch solche, die als Differenaerscheinungen zwischen alten (eingeübten, schwachbewußten) und neuen (neu vorgestellten, bewußtseinshellen) Willküräußerungen, bei Fortbestehen einer allgemeinen motorischen Willenstendenz während eines gleichzeitigen, nur temporären apperzeptiven Vorgangs, entstehen: sekundär ataktische Bewegungen (z. B. Versprechen, Vergreifen, Stolpern Erwachsener).

3. Ein Bestandteil der sekundär ataktischen Bewegungen ist stets eine sensorielle, der andere stets eine muskuläre Reaktion. (Dies diene zugleich als Kriterium bei einer Scheidung der ,assoziativen“ Ataxie von der organischen, z. B. bei Tabes.)

Z. f. d. g. Neur. u. Psych. LXXIX. 
4. Die primäre und die sekundäre Ataxie sind Abartungen in der Effektuierung derjenigen Vorstellungen, die den Bewegungswillen formen. Diese Störung ist eine ausschließlich assoziative, bei der die Kontinuität des psychischen und des physikalischen Vorganges prinzipiell völlig gewahrt bleibt; der Zustand 1. ist eine assoziative, der Zustand 2. eine dissoziative Ataxie.

5. Eine dissoziative Ataxie kann auch organisch bedingt sein. Beispiele: als Einleitung zu Apoplexie, bei organischen Veränderungen in der Hirnrinde (häufig, außer den sonstigen typischen Sprachveränderungen, bei Paralysis progressiva und bulbären Erkrankungen)."

Es sei hier daran erinnert, daß man mit Konsequenz zu vergessen pflegt, daß die Sprechbewegungen nicht nur Artikulationsbewegungen sind. In der Aphasieliteratur ist diesem Umstande in zu geringem Maße Rechnung getragen, die Wichtigkeit dieser Unterlassung wird in folgender Überlegung zutage treten.

Sprechen heißt: ich teile einem andern durch die moralisch-willkürliche Ausführung von Sprechbewegungen hörbar mit, was ich fühle und denke. Man vergißt, daß die Sprechatmung keine Stoffwechselatmung ist, sondern eine dem Sprechakt koordinierte, affektive. Man denke an die fast sprachlosen Idioten, die nur mit der Atmung sprechen: schnelles laut hörbares, eine Zeitlang fortgesetztes Atmen spiegelt in Verbindung mit Gesichtsausdruck und Körperhaltung die ganze Skala der Gefühle, Strebungen und Begehrungen, deren diese Wesen fähig sind. Die erste Sprechbewegung des Kindes ist nicht sein erster Schrei, sondern einige Zeit später das dem eben geschilderten in allen Punkten gleiche bzw. grundsätzlich ähnliche schnelle Atmen, z. B. bei Fixierung der Aufmerksamkeit auf einen starken Sinnesreiz in Verbindung mit einem unklaren und primitiven Begehren. Es sind das Ausdrucksbewegungen, von denen noch gar nicht genug studiert ist, ob sie beim einzelnen Kinde im Anschluß an Tasteindrücke, an Gesichts- oder Gehörsempfindungen zuerst auftreten (individuelle Vorstellungstypen), ob und welche Beziehungen zu Körperbewegungen, zu Mimik und Geste bestehen $u$. ä. Die später folgenden Intonations-, Phonations- und Artikulationsspiele und -übungen dokumentieren das Vorhandensein höherer Gefühle und stärker besetzter Vorstellungskomplexe; es ist das die Zeit, in der schon von Gedankenreihen gesprochen werden darf. Der auditiv regulierte Äußerungsdrang wird zu einem geordneten Mitteilungstrieb und erreicht bald die Stufe der Onomapoese. Hier finden die ersten Artikulationen statt, indem typische und starke Eindrücke von Dingen und Reizen mit primitiven Lautbildern symbolisiert werden (,Wau-wau“Sprache). Die Koordination der drei Abteilungen des Sprechapparates ist hier völlig vorhanden und wird zur Bildung der ältesten Sprachsymbole benutzt. Während des Übergangs zur Ausgestaltung eines 
reicheren Schatzes an Wortklängen der endgültigen Sprache setzt in vielen Fällen die (primäre) assoziative Ataxie ein. In diesem Stadium handelt es sich ausnahmslos um Ataxie im Bereich der Wortformung; die Phonation und die sprachliche Atmung sind nicht von Dissoziation betroffen. Das Wortklanggebilde ist symbolisiert, auch ist es als solches genügend scharf aufgenommen, aber es ist noch nicht vielseitig genug assoziiert, es wird vom Mitteilungstrieb noch nicht zeitlos erreicht. Die Wiederholung von Anfangslauten und selbst ganzer (auch mehrsilbiger) Worte sind das Bild stattfindender „Bahnungen“, und sie sind hinsichtlich des Äußerungstriebes nichts weiter als das. Je nachdem werden die Kinder von selbst sehr häufig auftretenden primären Dissoziationen gar nicht weiter schockiert; die festere Assoziierung der scharfen Wortklangbilder schreitet fort, und nach einiger Zeit ist der Besitz so vervollständigt, daß die neu aufgenommenen Klangbilder ohne ataktisches Übergangsstadium in den Gebrauch übergehen. Aber eine gewisse Zahl bleibt in noch näher zu schildernder Weise so lange ataktisch, daß das assoziative Gefüge des Denkens direkt und indirekt in Mitleidenschaft gezogen wird. Wenn wir nach der Ursache dafür forschen, warum eine Anzahl von Kindern übermäßig lange im Stadium der Ataxie verharrt, so muß angenommen werden, $d a ß$ bei diesen Kindern die Klangwahrnehmungen (niedere Klangempfindungen, unassoziierte Intervallempfindungen) so schwache Grundlagen für das höhere Vorstellen bieten, daß diese letzteren dem Reizverlauf keinen einheitlichen und ihn völlig aufnehmenden Weg bieten. Mit anderen Worten: die höheren Besitze sind in der „Veranlagung" besser vorgebildet als es für die sichere Einordnung der niederen und für die von diesen wiederum abhängigen Funktionen physiologisch notwendig ist. Diese Kinder geraten in tiefe Beschämungs- und Zornaffekte, die ganz erhebliche Gefühlsstärken erreichen; der Gesichtsausdruck verzerrt sich auch unabhängig vom Sprechen, und die diskoordinierende Kraft der Erregung ist so groß, daß selbst ältere Primärkoordinationen wie Gehen und Greifen mit dissoziiert werden können. Trotz Fortbestehens der sprachlichen Ataxie ebbt nach Wochen und selbst Monaten die Gefühlsflut zurück, und dann wird die Aufmerksamkeit wieder der Umgebung und ihrer Sprache zugewendet. Wird jetzt systematisch dafür gesorgt, das allgemeine Lern-, Seh- und Probierbedürfnis des Kindes wachzuhalten bzw. zu steigern, so wird sich in fast allen Fällen in einigen Wochen oder Monaten die Assoziation der Wortklangbilder vollziehen, und es ist nichts weiter zu befürchten. (Es sei ausdrücklich betont, daß zur festeren Assoziation der Wortklangbilder das intensive Spielen und Betätigen der Myo- und Stereopsyche von mindestens gleicher Bedeutung ist wie ein exaktes und phonetisch eindrucksvolles Benennen von Dingen und Tun, und zwar, wie nach dem eben Gesagten einleuchtend ist, nicht nur im Sinne 
einer Ablenkung, sondern weil eben der Anteil der Myo-, Ergo- und Eidopsyche eine festere und beziehungsreichere Verbindung des Wortbildes mit seinem begrifflichen Bestandteil bewirkt.)

In der Zeit der stärksten Schockierung der Psyche wird natürlich eine Irritierung der Phonations- und Atmungsbewegungen mit stattfinden; diese muß jedoch nach den geschilderten Verhältnissen und nach Analogie mit den gleichen Verhältnissen beim Erwachsenen als rein sekundür aufgefaßt werden (vgl. weiter unten).

Die primär ataktischen Erscheinungen treten auch in dieser ersten Entwicklungszeit nicht auf, wenn das Kind ohne besondere Anspannung der Aufmerksamkeit bzw. affektlos imitatorisch spricht, so z. B. gleichzeitig mit anderen, oder singt. Da sich die gesunden und stark apperzipierenden Kinder eigentlich dauernd in einem Zustand einer gelinden Hyperthymie befinden, so kommt dieser Zustand nicht oft dem naiven Beobachter zu Gesicht.

\section{II.}

Die primäre Sprechataxie, die erfahrungsgemäß geeignet ist, den Ur. sprung zu einer höheren Entwicklung, zu einem Dauerzustand abzugeben, kann nicht nur durch Verschiebungen in der sprachlichen Entwicklung entstehen; sie kann, neben einer assoziativen, auch eine dissoziative sein. Die dissoziative Form kann bei besonders dazu veranlagten Individuen (ererbte oder erworbene Psychopathie) hervorgebracht werden a) durch psychischen Schock, psychisches oder starkes physisches Trauma, ferner $b$ ) durch Commotio cerebri und c) durch hochfieberhafte Krankheiten. Die primären Erscheinungen sind durchaus sprachliche; die betroffenen Individuen werden nicht schlechtweg Angstneurotiker, sondern sprachliche Angstneurotiker.

Aus praktischen Gründen sei der Punkt b) zuerst abgehandelt. Wir müssen uns mit der in Hunderten von Fällen festgestellten Tatsache abfinden, daß durch Trauma und hochfieberhafte Krankheit eine, etwa tage- bis wochenlang währende Vorstellungsdissoziation mit daraus hervorgehendem ataktischem Sprechen verursacht werden kann. Wie in meiner ersten Arbeit werde ich auch diese Utberlegung auf der Grundlage der Kassowitzschen Nervenleitungstheorie durchführen.

Die Grundlage unseres Bewußtseins ist ein ausgedehntes System gebahnter Reizleitungen im Gesamtgebiet der zentralen Neuroplasmabahnen. Das Verbundensein von Reizleitungssystemen verschiedener Herkunft beruht einesteils auf der Verbindung der Leitungsbahnen als solchen, anderenteils auf der Kongruenz der in den betreffenden Systemen vorhandenen Reizschwellenwerte. Die Helle des Bewußtseins ist da am stärksten, wo die meisten Reflexvorgänge sich abspielen; dabei ist es für das Bewußtsein als solches und die Bewußtheit einer Vorstellung ganz gleichgültig, ob die die Vorstellung zusammensetzenden Empfin- 
dungselemente im nahen oder ferneren Gehirnabschnitt lokalisiert sind; diese subminimalen Unterschiede im Ablauf des Reizvorgangs sind im koordinierten Ablauf der Bewegungsvorgänge oder im vorstellenden Bewußtsein weder bewußt noch experimentell je zum Ausdruck gekommen. Im biologischen Sinne gibt es im Gehirn mehrere Arten von reaktionsbereitem Protoplasma: einmal dasjenige, das den am häufigsten vorkommenden Reizabläufen dient, das normal-schwellenwertige. Dann dasjenige, das einen Reizschwellenwert besitzt, der demjenigen des gebahnten am nächsten steht, sei es, weil es schon einmal von Reizen durchflossen war, sei es, daß es durch die Entwicklung der Gattung, der Art oder der Familie ,angeboren“ veranlagt zu denken ist. Endlich gibt es eine Gruppe vorgebildeter Bahnen, deren Protoplasma im Funktionalismus der psychischen Vorgänge noch von Reizen gewöhnlicher Stärkegrade nicht getroffen worden ist. Der Reizschwellenwert der zweiten Art ist um ein weniges höher als derjenige der ersten, derjenige der dritten um vieles. Wir müssen annehmen, daß die Reizschwelle im System der großen Reflexzentren in einem ganz bestimmten Verhältnis zu dem des psychischen Gebietes steht, desgleichen der des autonomen und des sympathischen Systems. Ein großes Gebiet reagibeler Substanz der ersten Art steht gegenüber der annahmsweise im Brennpunkt des Bewußtseins gerade stehenden Vorstellung in nur allgemein mitreagierender Beziehung, es ist, im psychologischen Sinne, der Bereich aller Ubergänge vom Bewußten zum Un- und Unterbewußtsein; wir vermögen uns aber weder psychologisch noch biologisch ein bewußtes Vorstellen ohne diesen Hintergrund zu denken, denn der denkende Mensch kann nur vermittels eines Bewußtseins vorstellen, man kann nicht eine aus einer einzigen Vorstellung bestehende Psyche haben. Eine Assoziation ist eine Bahnung von Besitz zu Besitz oder von Besitz zu Neu, oder umgekehrt. „Eine Assoziation oder ein assoziierter Reflex kommt dadurch zustande, daß zwei Reflexe oder Reflexbündel öfter zu gleicher Zeit oder rasch nacheinander aktiviert werden und sich infolgedessen gut gebahnte zentrale Verbindungen zwischen den beiderseitigen Bahnen herstellen. Ist dies aber geschehen, dann kann der kombinierte Reizerfolg von jedem der früheren Reizkomplexe oder auch schon von Teilen derselben ausgelöst werden" (Kassowitz). Die einen gebahnten Reizverlauf bedingende Umlagerung der Moleküle in der Nervenbahn vollzieht sich im allgemeinen durch die Einmündung eines adäquaten Reizes, im Verlauf der Kontinuität des Neuroplasmas, in die feinsten Verästelungen der zentralen Bahnen, in die „Elementargitter" (Kassowitz). Der Reizleitungsprozß selber besteht im oxydativen Zerfall des feinsten protoplasmatischen Netzwerks in den Elementarfibrillen. (Hiernach bedürfen wir für eine biologische Auffassung des Bewußtseins einer Auffassung im Sinne des mathematischen Funktionsbegriffes.) 
- Zu einem vorstellenden Bewußtsein gehört nun aber vor allem das Vorhandensein dreidimensionaler Vorstellungen; die Assoziationen der Bestände der Sinnesempfindungen bewirken dies. Koordinierte Bewegungen wiederum können nur zustande kommen, wenn die Bewegungsreize von eingeübten, mehr oder weniger unbewußt gewordenen Vorstellungen reguliert werden. Ich habe S. 525 meiner Arbeit eine "Geschichte des Wortes und der fertigen Koordination" rücksichtlich der Sprache gegeben und nachgewiesen, wie die einzelnen Bestandteile im Laufe der geistigen Entwicklung sich aufbauen und verweise darauf. Das Seelenganze, das uns hier als Ursprungsstätte der Koordination interessiert, besteht aus mehreren "Seelen“, insofern wir dem Bereich der motorisch-dynamischen, der visuell-skriptorischen und der auditivverbalen Sphäre je eine receptorische und eine effektorische Seite zuerkennen müssen. Während im ersten Verlauf der Geistesentwicklung die Besetzung der einzelnen Teile fast getrennt vor sich geht, verschmelzen in der späteren Zeit, mit der höheren geistigen Arbeit der Schule am meisten, die drei Gebiete so vollständig miteinander, daß es besonderer Untersuchungen bedarf, um den ursprünglichen Vorstellungstyp eines Menschen festzustellen. Auch hier finden Ausbildungen von „Merksystemen" und von Gewohnheiten und Geschicklichkeiten statt, die für die exakte Koordination nötig sind.

Von diesen Gesichtspunkten aus ist nun zu untersuchen, wie ein Schock, und zwar sowohl ein psychischer wie ein körperlicher, auf die Koordination einwirkt ${ }^{1}$ ).

Groeningen (Über den Schock, 1885) verdanken wir eine umfassende und gründliche Arbeit, deren wesentliche Ergebnisse wir uns für den vorliegenden $Z$ weck zu eigen machen können. Nach ihm ist der Schock eine durch heftige Insulte bewirkte Erschöpfung der Medulla und des Rückenmarks. „Man kann die auf sensible Nerven einwirkenden Reize hinsichtlich der resultierenden Erregung in vier Stufen bringen:

a) Die niedrigste Stufe ist unwirksam; die Erregung überschreitet nicht die Schwelle des Bewußtseins und ruft keinen anderen bemerkbaren Effekt hervor.

b) Die mittlere löst die adäquate Empfindung aus.

1) Dieser Abschnitt der Arbeit war schon vor dem Kriege in der Anlage beendet. Ich habe keine Veranlassung, rücksichtlich der im Kriege gemachten Erfahrungen von der hier gegebenen Auffassung des Schocks und der entsprechenden Erklärungen der zur Beobachtung gelangten Folgeerscheinungen abzugehen. Es ist von mir augenblicklich nicht erinnerlicher Seite der Versuch gemacht worden, die am Schock und seinen Folgen Erkrankten als nicht krank zu stempeln. Nach der hier gegebenen Reduzierung dieser Frage auf eine Anschauungsgrundlage dürfte dieser Versuch hier unberücksichtigt bleiben, ohne daB der Vorwurf der Unvollständigkeit erhoben werden könnte. 
c) Die starke verwischt die Feinheit der Qualitätsempfindung und läßt das Schmerzgefühl oder ein ihm verwandtes in den Vordergrund treten.

d) Die höchste vernichtet jede Empfindung, vorübergehend oder dauernd $\left.{ }^{l}\right) . "$

Das entspricht dem über die Nervenleitungsvorgänge Gesagten und nimmt sich, auf die in Rede stehende Untersuchung angewendet, folgendermaßen aus: Eine übermäßige körperliche Gewalteinwirkung ist einerseits eine solche, die der mechanischen Verletzung sehr nahesteht oder eine solche ist; dies ist besonders dann der Fall, wenn die eigentliche Schmerzempfindung dabei aufgehoben ist. Dem nervösen Elementargitter des Zentralnervensystems strömen dann mit einem Male derartige Reizmassen zu, daß, da es sich um eine so gut wie ausschließliche Ưberreizung der Sphäre der Tasteindrücke handelt, eine Verdunkelung des taktil-räumlichen Bestandteils der Vorstellungen eintreten muß, die eine Verminderung des Bewußtseins, besonders der Schmerzempfindung, und eine übermäßige Erregung des Gefühls bewirken. Wenn aber aus dem Assoziationsgefüge der Vorstellungen nicht nur, sondern aus einer großen Summe von Vorstellungen selber, der taktile Bestandteil einseitig herausgerissen oder ausgelöscht wird, so ist für das Vorstellen und die Erinnerung eine Unterbrechung der Kontinuität des Bewußtseins gegeben. Andererseits ist eine Gewalteinwirkung schon dann eine übermäßige, wenn ihre bloße Nachwirkung so lange anhält, da $B$ eine entsprechende Vorstellungsdissoziation eintritt, oder mit anderen Worten: die erwähnte Bewußtseinsveränderung ist mit einer Vorstellungsdissoziation untrennbar verbunden und wird großenteils durch sie bedingt. Damit ist gesagt, daß die Affektwirkung als solche einen Anteil an der Bewußtseinsauslöschung hat. Aber auch dies kann nicht einfach kausal aufgefaßt werden. Affekt ist im biologischen Sinne nicht nur eine Reaktion des gebahnten oder ungebahnten, psychischen Vorgängen dienenden Gebietes; da wir es mit großen Erregungsstärken zu tun haben, so ist mit den angegebenen biologischen Verhältnissen die Auffassung vereinbar, daß die in Rede stehenden übermäßigen Reizmengen, soweit sie nicht von tiefer stehenden Reflexzentren abgeleitet werden, das den psychischen Vorgängen dienende Gebiet sogar durchschlagen und bisher ungebahntes Elementargitter-Protoplasma bahnen. Damit läßt sich die Beobachtung in Verbindung bringen, daß nach großen Erschütterungen die geistigen Fähigkeiten, nachdem die unmittelbare Schockwirkung abgeklungen ist, eine gewisse Erweiterung erfahren können. Alles dies muß noch ergänzt werden durch die Überlegung, daß die zentralwärts geleiteten Reizmassen im Schock einen anderen Reizwert haben als derjenige ist, für den die bewußtseintragenden Rindengebiete anschlußbereit sind.

1) Diese Einteilung entspricht dem biologischen Grundgesetz Arndts. 
Das Bewußtsein wird also als eingeengt, verdunkelt und, hinsichtlich der eingestellten Vorstellungen, als mit Bruchstücken, d. i. mit dissoziierten Vorstellungen besetzt, gedacht werden müssen. Sowohl Vorstellungen wie auch Koordinationen werden hiernach durch den Schock zerstört bzw. gestört werden können; es sind nur graduelle Unterschiede, die zum Teil auf der Erregungsstärke selber, zum Teil auf der Dissoziierbarkeit des Protoplasmas und damit auch der von ihm getragenen Funktionen beruhen. Es liegt auch im Bereich der Möglichkeiten, daß protoplasmatischer Reizzerfall direkt unter der Einwirkung einer die Schädelkapsel treffenden Gewalt stattfindet; das würde in noch höherem Maße die dissoziative Wirkung erklären. Natürlich kann eine Elementarfibrille nicht mehr und nicht weniger wie die absolut mögliche, d. i. die physiologische, Reizstärke leiten; jede andere Vorstellung wäre den wirklichen Verhältnissen nicht entsprechend. Ausschlaggebend ist die Gesamtmasse der einwirkenden Gewaltreizung. Wenn diese den gewöhnlichen Grad der Reizmenge um ein Vielfaches übertrifft, so werden die leitenden Fibrillen, sowohl die für gewöhnlich leitenden wie die für den vorliegenden Ausnahmefall in Tätigkeit mitversetzten, zu einer so enorm schnell sich vollziehenden Zerfalls- und Restitutionstätigkeit gezwungen, daß die Phase der Restitution nicht genügend Aufbaustoffe für den nächstfolgenden Leitungsvorgang bereitzustellen vermag; die Folge davon ist ein Zustand, der als Ermüdungszustand bezeichnet wird. In diesem ist der betreffende Abschnitt außer Funktion, d. h. er schaltet für einige Zeit aus, und zwar sowohl hinsicht. lich seiner physiologischen wie seiner psychologischen Seite. Hierbei sei darauf hingewiesen, daß die meisten der einen Schock herbeiführenden Reize von Natur so beschaffen sind, daß ihre Massenwirkung nicht mit dem Moment des Einwirkens schon aufhört. Damit gehen Reizerscheinungen im Gebiet der Vasomotoren einher, die hier nicht näher erörtert zu werden brauchen; sie erklären sich aus den gegenseitigen Beziehungen, die zwischen den Gebieten der Hirnrinde und den tieferliegenden Gehirnabschnitten bestehen. Wenn das Bewußtsein zurückkehrt und die Mehrzahl der physiologischen Vorgänge wieder von in regelrechten Bahnen verlaufenden Leitungsabläufen besorgt werden, so sind auch die Bedingungen für eine ungestörte Koordination der Bewegungen innerhalb normaler Affektlage wieder gegeben.

Die psychopathische Veranlagung hat als eine ihrer Grundlagen eine gesteigerte Reaktionsfähigkeit imGebiet der höherenFunktionen. In einem die Sicherheit der motorischen Funktionsabläufe gefährdenden Maße ist die Leitungsfähigkeit der elementaren Bahnen im Gebiet höherer Vorstellungen vorgebildet. Im Psychischen bedeutet das, daß z. B. eine höhere Bewegungsgeschicklichkeit schon ohne eine im Vergleich zu normalen Kindern ausgiebige Anreicherung der primitiven Empfin- 
dungen und Erfahrungen sehr frühzeitig vorhanden ist; solche Kinder sind mit „Fähigkeiten“ geboren, über die man sich leicht viel zu früh freut. In diesen Fällen ist also derjenige Zustand höherer Reizleitungsgeschwindigkeit schon vorhanden, der bei anderen Fällen erst im Laufe der Entwicklung und Erziehung künstlich erzielt wird. Die Affekte erziehen den Menschen, nicht die Überlegungen. In geistigen Organisationen dieser Art bewirken schon Reize von ganz wesentlich geringerer Stärke einen Zustand von Schock, der im übrigen dieselben Eigenarten aufweist wie der rein körperliche. Dies muß ja auch nach Sachlage theoretisch angenommen werden.

Die Dissoziierbarkeit höherer psychischer Besitze durch Toxine beruht ebenfalls auf der Schädigung der nervösen Grundlage. Einzelne Krankheiten disponieren besonders zu solchen „Bahnungen“, wie sie in unseren Fällen beobachtet sind, so z. B. der Keuchhusten. Zwischen der dissoziierenden Wirkung von Trauma, Scheintod, Schlag, Stoß, Eisenbahnunfall, Kitzeln, Ängstigungen, Meningitis, Encephalitis, Eklampsie, Typhus, Magendarmkatarrh, Diphtherie, Masern, Scharlach, Keuchhusten, Pleuritis, Pneumonie, Würmern und Vergiftung besteht ein verhältnismäßiger, aber kein grundsätzlicher Unterschied. Im späteren Verlauf des Leidens kommen häufig Zustände von Besserung oder Verschlechterung vor, die von äußeren Geschehnissen nicht abgeleitet werden können. Das hat seine Ursache einmal darin, daß zahlreiche Kranke zu den zirkulär und periodisch Verstimmten aus endogener Ursache gehören, wie es ja feststeht, daß zahlreiche Psychopathen auch ohne die hier vorliegende assoziative Störung Periodiker, Maniker oder Melancholiker in kleinem Ausmaße sind. (Die assoziative Sprachstörung habe ich nicht selten mit derartig hochgradiger Erschöpfung verbunden gesehen, daß man geneigt, wenn nicht gar genötigt war, Eisen und Lecithin gegen die Zeichen hochgradigen Gehirnverbrauchs zu geben.)

Eine posttraumatische Vorstellungsdissoziation ist der psychologische Ausdruck für eine wesentliche Veränderung des Schwellenwertes in den betroffenen Abschnitten. Das bedeutet aber für den koordinierten Ablauf der abhängigen Bewegung eine Aufhebung der vorher vorhanden gewesenen einfachen Verhältnisse, an deren Stelle eine Uneinheitlichkeit des Reizverlaufes nach der motorischen Seite hin tritt. Ein solcherZustand ist aber gleichzeitig im höchsten Maße geeignet, die Selbstbeobachtung auf die scheinbar verlorengegangene Funktion zu richten. Die mit den Fehlleistungen untrennbar verbundenen ungewöhnlichen Empfindungen repräsentieren im Bewußtsein den Begriff einer Störung, und der Beobachter kann nicht anders als die gleiche Bezeichnung anwenden.

Die auf diese Weise hervorgebrachte primäre Sprechataxie betrifft in der überwiegenden Mehrzahl der Fälle die Wortbildung, aber auch die Tonbildung und die Sprechatmung. 
III.

Die Schocks (einmalig, mehrmalig oder fortgesetzt) können allgemein durch Bahnung eine graduelle Veränderung der nervösen Organisation hervorbringen. Da aber eine Anzahl nervös minderwertiger bzw. latent nervöser Konstitutionen nach solchen Schocks (nach sehr geringen Reizstärken) gerade primär sprachlich dissoziiert werden, so muß gefolgert werden, daß eine besondere Geneigtheit zu sprachlichen Dissoziationen einen für sich charakteristischen Labilitätszustand gewisser nervös minderwertiger Konstitutionen ausmacht.

Aus dem Anblick des bunten Bilderreichtums der nervösen Entartungszustände erhellt, daß sie unter anderen Erscheinungen die Eigenart großer Veränderlichkeit der Vorstellungskomplexe darbieten. Diese Veränderlichkeit ist eine sowohl qualitative wie auch quantitative; es können, vorzugsweise durch Affekte bedingt, sowohl zahlreiche als auch große Vorstellungskomplexe ,gesprengt" und verändert werden, vom einfachsten Werturteil angefangen bis zum Ich-Bewußtsein. Keineswegs ist eine solche Konstitution am Ende ihrer Veränderlichkeit angelangt, wenn gewisse primäre Dissoziationen stattgefunden haben; gerade die affektiv stark bewegte Psyche produziert, und über den produzierten Abartungen der Urteils- und Wissensbestände (die Störung geht bekanntlich bis zur Gedächtnisumwertung, wie bei der Pseudologia phantastica) gerät das Individuum in eine solche Stellung zur Mitwelt, daß aus den alltäglichen Ereignissen Schock auf Schock entsteht und seinerseits weiter wirkt. Aber gerade die Kategorie der sprachlich Dissoziierten hebt sich scharf ab von der großen Menge; sie sind gewissermaßen die auffallendsten, gröbsten, obwohl man gemeinhin nur die expressive Seite der Anomalie wertet. Schon aus diesen Gründen ist zu fordern, das gesamte Gebiet der durch ,Stottern“ (die fortbestehende Form sprachlicher Dissoziationen) charakterisierten Zustände unter der Bezeichnung „,assoziative Aphasie“ zu begreifen und zu systematisieren.

Die Gefahr einer Unklarheit der Abgrenzung gegenüber den Sprachverwirrungszuständen ist nicht zu befürchten. Die verwirrte Sprache ist die expressive Seite eines verwirtten Denkens; die eigentlich sprachlichen Assoziationen (Koordination der sprachlichen Atmung, Phonation und Artikulation, Symbolie, verbal-begriffliches Vorstellen) sind nicht dissoziiert. Die Sprachstörungen der Paralytiker sind, obwohl als organisch bedingte hier nicht besonders abgehandelt, ebenfalls assoziativ im Sinne der primär ataktischen Störungen; das gehäufte Verschreiben und Versprechen ist ein Zeichen dafür, daß, wenigstens im Anfang, die Störung oberhalb des expressiven Gebietes liegt. Gegen den Ausdruck ,,assoziative Aphasie“" könnte eingewendet werden, daß es sich nicht um eine Aphasie im wortwörtlichen Sinne handele, weder bei der Paralyse noch bei den seither unter "Stottern" begriffenen 
Störungen. Um einen eigentlichen Verlust handelt es sich aber bei den wenigsten der bisher unter der Sammelbezeichnung Aphasie begriffenen Störungen, ob auf organischer Grundlage oder nicht. Die Relativität des Sprachverlustes ist ja eben durch das Wort ,assoziativ" in der richtigen Weise gekennzeichnet. Keineswegs ist es überflüssig, gerade auf diesem Gebiet die Wesensart der Störung neu zu charakterisieren; das Beharren bei der volkstümlichen Bezeichnung hat in größtem Umfange, und lange genug, zu Stillstand und Unklarheiten geführt.

Nun wirft aber das Vorhandensein einer streng abgegrenzten Geneigtheit einer nervösen Organisation zu primär sprachlicher Dissoziation die Frage nach der Art und der Ursache dieser Geneigtheit auf. Nach dem, was wir vom Wesen des zentralen nervösen Leitungsvorgangs, im Besonderen und hinsichtlich des Bewußtseins, wissen, müssen wir eine gewisse Zeit - oder eine gewisse Höhe des Leitungswiderstandes oder des Reizschwellenwertes - für die Dauer eines Reizleitungsvorgangs fordern, wenn im Bewußtsein ein entsprechender und exakt ablaufender (paralleler) psychischer Reflexvorgang erfolgen soll. Oder mit anderen Worten: das Wortklangbild muß wirklich unter Erfüllung aller Vorbedingugen (nämlich bei vorhandenem Besitz an Empfindungen derTonhöhenunterschiede, der Intervalle, der Klangfarben usw.) bewußter Besitz sein oder gewesen sein und, ohne durch geringfügige Affekte auslöscht zu werden, klar vorgestellt werden können, wenn seine effektorische Seite, der gesprochene Wortklang, von sicherer Körperfunktion getragen sein soll. Es bedarf einer nur minimalen Herabsetzung des Reizschwellenwertes z. B. der zentripetalen Gehörsleitung, um sofort eine Unsicherheit der Reflexvorgänge der effektorischen Seite, einen Ausfall oder eine Koordinationsstörung hervorzubringen. Man käme so zur Konstruktion des Hilfsbegriffes einer "Neurasthenia acustica". Das würde zur Erklärung der hier interessierenden Vorgänge nicht genügen; denn eine „Asthenakusmie“ (analog der Asthenopie), die man praktisch übrigens sehr wohl auf eine Anzahl von Fälle anwenden kann, würde nichts weiter als gelegentliche Beschleunigungen der Perzeption bewirken; stellt man sich aber vor, daß die Beschleunigung des Reizverlaufs noch etwas weiter oberhalb hinaufreicht, so sind Koordinationsbeeinflussungen unvermeidlich. Damit aber der Störungsvorgang ins vorstellende Bewußtsein gelangen und dort abnorm schnell festgehalten und assoziiert werden kann, muß gerade die neuro-psychopathische Konstitution als Hintergrund dazukommen. Das erklärt auch das Ausbleiben sekundär-assoziativer Verknüpfungen bei in erwachsenerem Alter vorkommenden vorübergehenden Sprechataxien. Eine Herabsetzung des Reizschwellenwertes im Gebiet der Klang-, Intervall- usw. Empfindungen kann auch bewirken, daß die zentripetalen Erregungen in untergeordneten Zentren (z. B. Vierhügelsystem) abgeleitet werden 
und im Sprachgebiet eine nur noch ungenügende Stärke besitzen; oder man kann umgekehrt annehmen, daß im Schock oder im Affekt auch aus den autonomen Systemen abnorme Reizstärken zum Sprachgebiet durchgeleitet werden. Hiervon haben wir keine bewiesenen Vorstellungen $^{1}$ ). - Ich nehme an, daß auch eine besonders günstige Bahnung der effektorischen Seite des Sprachvorganges, in unserer Kulturschicht besonders, vorliegt: eine „Neurasthenia motorica“ des Sprachgebietes bei den zu „Stottern" Veranlagten, eine angeborene größere Leichtigkeit für die Spracherlernung einschließlich ihrer effektorischen Seite allgemein. Dafür sprechen manche Fälle von sog. ,PPoltern“, das mit dem ,Stottern“ grundsätzlich verwandt ist. - Die angenommene Herabsetzung des Reizschwellenwertes im perzipierenden Abschnitt könnte ungezwungen als eine „Bahnung“" durch die übermäßige Geräuschmasse des Kulturverkehrs (man vergegenwärtige sich die wohltuende Stille unzivilisierter Länder) erklärt werden ${ }^{2}$ ). Für die Annahme, daß in früheren Jahrhunderten Affekte im vorliegenden Sinne sprachdissoziierend gewirkt haben können, spricht der Umstand, daß z. B. Stotternheim bei Erfurt und Stammersfelde in Pommern in Gegenden liegen, in denen sich zur Zeit desDreißigjährigen Krieges große geschichtliche Vorgänge abgespielt haben ${ }^{3}$ ).

Gemeinsam ist allen ähnlichen Zuständen, daß die schädigende, die primäre Dissoziation herbeiführende Noxe entweder einen vermehrten Reizbarkeitszustand der zentralen Bahnen zurückläßt oder einen solchen schon, als Ausdruck konstitutioneller Minderwertigkeit, vorfindet, und daß nun diese drei Faktoren: das Trauma (als Erinnerungsbild bewertet), die primäre Koordinationsstörung (die primäre Ataxie, die oft selbst erst das primäre schockierende Trauma ist) und die schockierte Psyche einen Circulus vitiosus zu zweit oder zu dritt bilden, den die psychopathische Veranlagung endlos unterhält und ausbildet.

Hiernach muß es als aussichtsvoll erscheinen, von einer Klinik des „Stotterns" bzw. der assoziativen Aphasie zu sprechen, also nicht nur von Ursache und Erscheinungen, sondern auch vom Verlauf. Es liegt im Wesen der durch Funktionsstörung herbeigeführten assoziierten Vorstellungskomplexe, daß sie nicht schlechthin ein Fremdkörperdasein führen, sondern eben ein solches in einem lebendigen, sozial beziehungsreichen geistigen Ganzen, das mit zunehmendem Alter Anpassungen

\footnotetext{
1) Manche Vagus-Erscheinungen bei „Stotterern“" sprechen dafür.

2) Die Häufigkeit der syphilitischen Neurorezidivs VIII. verdient in diesem Zusammenhang Erwähnung.

$\left.{ }^{3}\right)$ Die zahlreichen Schock-Erkrankungen des Krieges sind „Bahnungen“. In den wohl zahlreichsten Fällen lag die Menge der Bahnungen nicht auf dem Ge. biet des Gehör-Sprach-Sinnes, sondern auf dem des Körperempfindungs-Bewegungssinnes. - Wenn man auf Grund der Theoreme Storchs und Heinrich Sachs' eine psychophysische Darstellung des Bewußtseins im Raume versucht, so wird das Gesagte ohne weiteres klar. Davon an anderer Stelle.
} 
und Selbsthilfen produziert. Diese Produktionen erfolgen, je nachdem das Erinnerungsbild des ursächlichen Traumas, oder das primär ataktische Sprechen, oder die „Persönlichkeit" den Mittelpunkt abgeben.

IV.

Die Tatsache, daß der Sprechende eine Rekonstruktion des durch primäre Sprechataxie ihm als gestört zu Bewußtsein kommenden Wortklangbildes unternimmt, ist durch Beobachtung, Exploration und Analyse festzustellen. Eine Diskussion dieser These ist von der Diskussion des Verhaltens der Sprachvorstellungen nicht zu trennen; diese hat vielmehr vor der Zulassung anderer Erklärungsversuche aus Gründen der psychophysiologischen und biologischen Denkweise zu erfolgen.

Ich habe in meiner Arbeit „Stottern als assoziative Aphasie“" gerade im 3. Kapitel die ausführliche theoretische Darlegung der Leitungsverhältnisse gegeben und beziehe mich darauf, besonders auf Seite 492 bis 495; wiederholt seien folgende Punkte:

1. „Eine affektive Sprengung von Vorstellungskomplexen findet als normale Möglichkeit statt. Diese muß bei Menschen mit psychopathischer Veranlagung besonders häufig sein (,reizbare Schwäche"), ist also sowohl funktionell wie charakterologisch wichtig.

2. Damit ist die Möglichkeit gegeben, daß Simultaneinstellungen affektiv, vorübergehend, eintreten können.

3. In diesem Falle entsteht eine Koordinationsstörung, die ataktische Bewegungen hervorzubringen imstande ist (vgl. Kapitel 2).

4. Ein Widerstreit von Kräften erzeugt nie Spasmen, sondern nur Ataxie, die wir in diesem Falle (im Gegensatz zur primären Assoziation) als sekundär dissoziativ bezeichnen müssen (vgl. Laubi). Beispiele aus dem täglichen Leben sind hinreichend vorhanden (Verlegenheitsstolpern, Erregungsstottern, Versprechen, Vergreifen; ähnlich bei Freud).

5. Auch die neurologische Betrachtungsweise bei der Frage nach dem Ursprung der Stotterbewegungen führt lediglich zur physiopsychologischen Betrachtungsweise des Begriffes der Koordination.

6. Nur wenn wir uns das Verhalten des Reizverlaufs nach Kassowitz klarmachen, können wir die vorkommenden Fälle von Vorstellungsdissoziation begreifen, also alle die Fälle, in denen sich Stottern nach Infektionskrankheiten und Schock entwickelt.

7. Die Untersuchung der Koordination hat nunmehr ausschließlich eine solche zu sein, die die grundsätzlichen Assoziationsmechanismen feststellt (vgl. Kap. 4).

8. Das ausgebildete Stottern darf, ohne daß man dabei an einen lokalisierten Defekt zu denken hat, von Gesichtspunkten der Aphasielehre aus behandelt werden. Man würde hiermit eine einheitliche Vorstellung vom eigentlichen Wesen der Krankheitin die Literatur einführen." 
Hiernach können weitere Betrachtungen in dieser Richtung vorgenommen werden. Die Aufmerksamkeit des Sprechenden bemächtigt sich, wie die Beobachtungen an allen geeigneten, d. h. beginnenden Fällen lehren, der Erscheinungen der primären Sprechataxie sehr bald. In späteren Stadien wird jedoch, wie ausdrücklich betont sei, als eins der vielen Mittel, die Seele von den Konflikten frei zu halten, die sich aus Stottern vor zuhörenden Menschen ergeben, die Ableugnung der Tatsachen betrieben. Je nach der Veranlagung des (primär) Stotternden tritt entweder Beschämung, Verlegenheit oder Ärger, also ein Affektzustand, oder eine Anzahl frühzeitiger Selbsthilfsversuche (also eine Reflexion) zum Sprachäußerungswillen hinzu.

Nachdem die Natur der primären Sprechataxie als assoziative oder dissoziative Koordinationsstörung nachgewiesen ist, stehen wohl keine Bedenken entgegen, in der affektiven oder reflexiven Erregung der Psyche die Ursache zur Betätigung der Aufmerksamkeit zu sehen. Hierfür ist die Grundlage gegeben in der Perzeption der sich als Sinneseindrücke dem Bewußtsein aufdrängenden Störungsempfindungen. Diese sind, um das vorwegzunehmen, gewissermaßen phylogenetische Rück. schritte. Die Sprechbewegungen sind, in dem Alter, in dem sich das (primäre) Stottern einstellt, schon reine Lautierbewegungen; der Reichtum an lebhaften und zur Außenwelt bezogenen Vorstellungen ist schon ein so großer, daß von einer Blütezeit der sprachlichen Symbolie gesprochen werden muß. Jede Lautprägung wird nicht nur bewußt und unbewußt auf ihren Gleichklang mit den Klangbildern der Erwachsenen geprüft und geformt; die Erwachsenen werden hinsichtlich ihrer Handlungen genau beobachtet, und aufs schärfste wird untersucht, wie sie auf diese oder jene Lautbildung hin ihr Tun und Lassen einstellen. Es besteht schon ein wertvoller und reichhaltiger Schatz von Wortgebilden, der einen großen Teil der springlebenden kindlichen Äußerungstriebe, Strebungen und Begehrungen verkörpert, wenn das primär ataktische Sprechen einsetzt. Häufig merkt der Beobachter, daß die Zeichensprache in Gestalt von Mimik und Geste wieder stärker mitbenutzt wird, wenn die ersten Störungen einsetzen, abgesehen von geradezu erschütternd starken Beschämungen und starren Wachträumereien, hinter denen sich das Nachdenken über die rätselhafte Erscheinung verbirgt. Jedenfalls geht schon aus diesen wenigen Feststellungen hervor, da $B$ eine Anzahl innerer Bedingungen erfüllt sind für die Perzeption und Apperzeption der Störungsempfindungen, die mit dem primär ataktischen Sprechen verbunden sind.

Diese Störungsempfindungen sind vorzugsweise taktil-motorischer Art; natürlich zugleich akustischer Art, aber das erstere ist das auffallendere, weil es dem Prinzip entgegengesetzt ist, nach dem das Sprechen gelernt und beobachtet wurde. Es handelt sich hier um das Wenn der 
ganzen Sache (nicht der Auffassung): wem sich das Erwähnte aufdrängt, der betritt eine grundsätzliche Entwicklung - wem nicht, der betritt sie eben nicht, und es tritt Vergessen ein. Man kann mit ziemlicher Sicherheit sagen, daß diejenigen primär Stotternden, denen sich das taktile Element (die verlängerte oder verstärkte oder wiederholte Berührungsempfindung, oder die des Widerstandes, der Spannung, des Vorstellens der ungewohnten Stellung im Raum) aufdrängt, vorzugsweise die typischen (und häufigsten) sogenannten Konsonantenstotterer werden (man fühlt das Dillettantische und Verzerrte dieser Bezeichnung, die sich in manchen Kreisen immer noch einer durchaus unberechtigten Pflege erfreut), während diejenigen, die das gestörte Klangbild erfassen, und infolge der größeren Schwierigkeit, mit Bewußtsein zu phonieren, die sprachlich von den ersteren stark verschiedenen Vokalstotterer abgeben; ein Ausbau dieser Unterscheidung ist jedoch durch die wirklichen Verhältnisse nicht begründet.

Jetzt tritt also der Zustand ein, in dem der Ausdruck "Stottern" nach wissenschaftlicher Feststellung jede Spur von Berechtigung verliert: die Störung geht aus dem zufälligen in den ,ppervers-willkürlichen“" über. Selbstverständlich ist auch die primäre Ataxie nicht zufällig; es liegen ihr eben Störungen der Assoziationen zugrunde, deren Grundsätzlichkeiten abgehandelt sind; aber sie verschwindet fortan mehr und mehr unter der Einwirkung der Willküren, die vom Sprechenden zum Zweck der Wiederherstellung des ungestörten Sprachablaufs bzw. der Wortklangbilder ersonnen und ausgeführt werden. Der Sprachablauf wird aber im Falle primär ataktischen Sprechens nicht nur wegen des Auftretens sinnlich wahrnehmbarer Störungsempfindungen als gestört empfunden; das muß ausdrücklich betont werden, denn sonst bleiben eine Anzahl primärer Selbsthilfen unerklärlich, und es könnte scheinen, als ob hier ein Punkt gegeben wäre, die ganze Anschauung aus den Fugen zu heben. Darum sei schematisch zusammengestellt, welche Faktoren der Sprach. koordination tatsächlich gestört werden und als gestört zu Bewußtsein kommen können:

I. Sinnlich: Hervortreten der Qualitäten des Tastsinns:

1. Berührungsempfindungen (Oberflächensensibilität).

2. Spannungsempfindungen (Muskelempfindungen).

3. Stereovorstellungen.

4. Gelenkempfindungen.

5. Empfindungen in Kehlkopf, Bronchialstamm und Thorax, die sonst noch nie zu Bewußtsein gekommen sind: Artikulationsempfindung der Glottis, bewußte Innervation der Intercostales (diese ist sonst unbewußt; jeder Arzt weiß, daß kein Kind begreift, was man von ihm will, wenn man sagt: „tief atmen!"), Luftdruckempfindung im Bronchialstamm. 
6. Die Klangwahrnehmung der wiederholten, neu und fremdartig ausgeführten Lautgebilde; diese haben den psychologischen Wert zunächst unassoziierter Klangempfindungen. (Vgl. meine Arbeit: Schema zur Geschichte des Worts usw., S. 525.)

II. Sprachlich: Die ataktisch hervorgebrachten Lautgebilde sind außerhalb der Sprachsymbolie stehend; daher

1. „Fremdkörper"-Lücke im Verständnis des Selbstgesprochenen.

2. Unlustgefühl über die verletzte Symbolie des Klangbildes.

3. Zeitliche Verzögerung des Sprechvorganges gegenüber der weitergesprochenen inneren Sprache.

4. Unlustgefühl über den häßlichen Wortklang.

III. Psychisch: 1. „Ich kann nicht“ kommt als Werturteil zu Bewußtsein, oft nur ganz allgemein.

2. Durch Verzögerung und Versagen der Funktion entsteht eine Erkenntnis der Fehlleistung, psychologisch gleichwertig der Vorstellung von Krankheit oder Dummheit.

3. Spaltung des Gefühls. Primär war vorhanden: ein aus positivem Gefühlston resultierter Äußerungstrieb; nach dem Bewußtwerden der Fehlleistung: Triebhemmung durch Unlust: Grundlage der "Angst“" (,Pseudoschizophrenie" ").

4. Verlagerung der Aufmerksamkeit: a) sozial: vom Objekt (dem Angeredeten) zum Subjekt (dem Sprechenden selber);

b) sprachlich: von der inneren Sprache zum Sprechvorgang, und zwar: entweder sensorisch (zur Dissoziation) oder motorisch (zur Ataxie).

Es liegt in der Natur der Sache, daß dies Schema aus der rein beschreibenden in die entwickelnde Form übergeht.

Die hier gegebene Ubersicht wird leichter verständlich, wenn man bedenkt, daß dem Kinde im Alter der primären Sprachdissoziation nur geringe Abstraktionsfähigkeit innewohnt. Seine Sprache ist naiv wie sein Handeln; sie hat Nützlichkeitswert, aber noch keinen ästhetischen oder Selbstzweck, sie ist ein sehr einfaches Lautwerden sehr kurz angebundener starker Triebe, Strebungen und Begehrungen. Erst in der Schule bekommt sie Selbstzweck; sie dient zur Klärung der Begriffe, sie bekommt, durch die Zensierung der Denkleistung, eine neue und starke Betonung ihres persönlichen, sozialen und äußeren Wertes. Aus diesen Gründen wird die Aufmerksamkeit planmäßig auf sie gelenkt; ihre ästhetischen wie auch, beim Erlernen fremder Sprachen, ihre Bewegungswerte treten vor die Selbstbeobachtung; Leistung und Fehlleistung bekommen eine gewissermaßen kritische, um nicht zu sagen einseitige Zugehörigkeit: was das Kind auf dem Spielplatz als richtig, selbstverständlich und unbewußt besaß, erwirbt es zum zweiten Male, denn seine coram publico vorgetragenen Schulantworten gehören teils ihm, teils einem Dritten (dem Lehrer), und der Begriff des „richtigen“" 
Könnens wird zu pedantischer Schärfe erhoben, der auch bei Sprechgesunden manchen Angsttraum (bei Tage und bei Nacht) hervorbringt. So ,verschlechtern" sich die assoziativen Sprachstörungen in der Schule aus begreiflichen Gründen, denn die Bedingungen für die Anreicherung sekundärer Assoziationen sind durch innere und äußere Beziehungen der Person und der Leistung so günstig wie möglich. Ganz zu schweigen von den mit völlig unzureichenden Kentnissen unternommenen Versuchen der. Lehrenden, verbessernd einzugreifen.

So kommen die Einzelelemente der Dissoziation auch nicht alle sofort zu Bewußtsein, sondern erst nach und nach; um so früher, je größer die vorhandene Geneigtheit zu Selbstbeobachtung und die Fähigkeit zur Abstraktion, je lebhafter die höheren Gefühle erregbar sind, und je größer die Geneigtheit zu negativen Gefühlen und Affekten. Utberdies ist stets daran zu denken, daß die Lebhaftigkeit des Äußerungstriebes (s. u.) die Hauptursache für die Assoziationsmöglichkeit überhaupt ist.

Die Störungsempfindungen werden gerade bei Kindern mit dieser Veranlagung oft überraschend schnell zu bewußten Störungsvorstellungen. Sie haben den psychologischen Wert von Kontrastvorstellungen.

Der Begriff der Symbolie soll hier nicht erörtert werden, so nahe es auch liegt, und so günstig die ganze Materie auch dazu ist. Es soll nur gesagt werden, daß das Gefühl für Ähnlichkeit, Gelingen und optimalen Kraftverbrauch bei erfolgreicher Leistung ein weithin wirkendes Lustgefühl ist, das sich den Äußerungstrieb zu freier Betätigung einstellen läßt, und daß die Einbeziehung vorgebildeter Bahnungen in den psychischen Reflexvorgang nicht nur eine Verbindung mit nahegelegenen, sondern auch mit fernerliegenden ähnlichen Werten bewirkt. Hierdurch aber wird ein ,,Ähnlichkeitsfeld“" geschaffen, in welchem in viel weiterem Umfang Lust oder Unlust bei Ähnlichkeit oder Unähnlichkeit auftritt, als dies nach den bloßen Vorstellungswerten im Sinne der Assoziation zu erwarten wäre. In diesem Sinne ist der Punkt 1 der Gruppe II von besonderer Wichtigkeit. Die wiederholten Buchstaben- und Silbenansätze der Wortanfänge, die den dissoziativen Charakter der Erscheinung bezeichnen, sind nicht gesprochen (im Bereich der inneren Sprache "gedacht"), sondern eben physiologische oder mechanische Ergebnisse einer Spaltung, einer Hemmung, sind „Zutaten“. Wenn ein Kind den Anfangsbuchstaben des Wortes „David“ ataktisch ausspricht, so läßt sich das weder schriftlich noch mündlich vom beobachtenden Refer enten anders wiedergeben als mit dem bekannten Sprachlaut bzw. grammatikalischen Wortbestandteil ,d ", und in der entsprechenden Ausdrucksweise. Es muß nachdrücklich darauf hingewiesen werden, da $B$ der Beobachtende und auch der Sprechdissoziierte, wenn sie schon lesen und schreiben gelernt haben, nicht anders können, als den wieder- 
holten Artikulationsbewegungen bzw. dem während der Ataxie entstehenden Lautgebilde den psychologischen Wert eines Buchstabens beizulegen. Hierzu liegt aber keine Berechtigung vor; denn ein Kind von 4-5 Jahren weiß noch gar nicht, was ein Buchstabe ist. Wenn ihm die Erwachsenen in ihrer Weisheit nicht die Suggestion geben, daß es sich um Buchstaben handelt, so kommt das Kind von selbst noch lange nicht. darauf, und der Begriff des gestörten Sprechens bleibt bis auf weiteres repräsentiert durch die Empfindung bzw. die Vorstellung einer Berührungs-, Spannungs- oder Druckempfindung, einer Lautwiederholung, und folglich durchaus nicht so eng assoziiert, nicht so verhängnisvoll bezogen wie im Falle der autoritativen Benennung. Diese Denkweise wird bei vielen dissoziiert Sprechenden durch Befragen ermittelt werden können. Die genannten Empfindungen liegen zunächst außerhalb der inneren Sprache, da sie unbewußt, nicht vorgestellt entstehen; sie bleiben es je nach dem Grade der psychopathischen Alterierbarkeit der Gefühle kürzere oder längere Zeit; dann aber werden sie, besonders durch nachfolgende Schockierungen (Aufmerksammachen, Verlachen, Strafen (!) und auffallende Blamagen), in noch näher zu schildernder Weise energisch assoziiert, d. h. dem Assoziationsgefüge der inneren Sprache, dem Denken in Worten, einverleibt, und zwar sowohl die Vorstellung der Störung wie das auftretende Unlustgefühl; der Übergang besteht darin, $\mathrm{da}$ die durch eigenes wie durch fremdes Urteil zu Sprachwerten symbolisiert werden. Aufmerksamkeit und Selbstbeobachtung, Reflexion und Erlebnisse wälzen von nun an einen gewaltigen Ballast sprachlicher Sekundärassoziationen vor die Bresche der primären Dissoziation, damit. dem Sprechakt seine soziale und psychologische Vollgültigkeit gerettet wird.

Die Bildung bzw. das Auftreten von Unlustgefühlen ist besonders hervorgehoben. Nicht weil es nicht selbstverständlich wäre, sondern weil diese Unlustgefühle, wie alle Gefühle, vorgestellt werden können; damit ist die Grundlage gegeben für die ängstlichen Erwartungsaffelkte der späteren Zustandsbilder. Gerade durch diesen Umstand, daß auch lebhafte Unlustgefühle sich mit den ataktischen Wortanfängen verbinden, wird bewirkt, daß die ganze Gruppe der sekundären Assoziationen (der Störungsvorstellungen) zu einem einheitlichen und stark bezogenen Vorstellungskomplex wird. Das aber bedeutet eine zunehmend starke Blockierung des sprachlichen Äußerungstriebes. Dies hat eine große Ähnlichkeit und nahe Verwandtschaft mit dem dauernden Vorstoßen und Festgehaltenwerden unseres Triebes nach assoziativer Entspannung und Kongruenz, die wir erleben, wenn ein uns bekanntes Tonstück schlecht gespielt wird (d.h. mit Abbrechen, Wiederholungen und Fehlern).

Noch eine wichtige Feststellung sei hier eingeschaltet. Die Utbergänge von einem druckreifen akademischen Sprechen, das alle Vorzüge der Logik wie der Aussprache besitzt, zu einem mehr oder weniger 
temperamentvollen, mehr oder weniger ungrammatischen und begrifflich unscharfen, ungesammelten Daherreden sind sehr zahlreich. So gibt es auch unter den Sprachdissoziierten Vertreter jeder dieser beiden extremen Gruppen sowie aller Spielarten. Man muß hier, um den eigentlichen Sprechtypus festzustellen, scharf trennen zwischen dem Sprechen ohne den Einfluß des Störungsvorstellens und mit demselben; erst nach Ermittelung dieses Typs tritt z. B. bei der betreffenden Gruppe die eigenartige „Verschiefung des Denkens" hervor, die (s. u.) entweder einen assoziativ-aphatischen Zustand aufweist, der völlig an sensorische Aphasie erinnern kann, also zum assoziativ-aphatischen Zustand gehört, oder der psychopatischen Konstitution primär eigen ist.

Die ganze Gruppe der unter III erwähnten Störungsvorstellungen wird weiter unten abgehandelt werden.

Man wird anfängliche Bedenken gegen die Auffassung, daß diese relativ abstrakten Vorstellungen in jungen Jahren gewonnen sein sollen, nicht ganz unterdrücken können. Aber es handelt sich auch nicht darum, für alle Fälle kindlicher Sprachdissoziation diese Abstraktionen gleich auf den alleranfänglichsten Zustand anwenden zu sollen; die Darstellung der Materie verlangt nur einen möglichst frühzeitigen Hinweis auf die sich alsbald vollziehenden Ausbauformen. Das etwa zu fehlen Scheinende wird an geeigneterer Stelle zu finden sein. An und für sich können die gewonnenen Ergebnisse jederzeit nachgeprüft und demonstriert werden. Überhaupt ist vieles nur im täglichen Umgang mit den Patienten zu beobachten.

Das gilt besonders für die Anerkennung der für das Verhalten der Aufmerksamkeit ausgesprochenen Gründe. Man erkennt gerade bei den Beobachtungen an Sprachdissoziierten, wie durch auffallende Empfindungen und verlagerte und gereizte Gefühle die Merkfähigkeit hierfür gesteigert und erweitert wird, wie durch Anreicherung des assoziativen Bestandes die Beziehungen sich grundsätzlich festlegen und vervielfaltigen, wie die Aussaat selbständiger Produktionen stattfindet und das Gebiet der Störungsvorstellungen sich dem assoziativen Aufbau des Bewußtseins und Selbstbewußtseins eingliedert und Eigenbenennung und Eigenleben gewinnt.

Der Schwerpunkt für die weitere Entwicklung der geschilderten Verhältnisse liegt in dem Fortbestehen der Ursachen für wiederholte Ataxien. Denn es hat eine völlig in den Grenzen der psychologischen Geschehnisse sich abspielende Substitution stattgefunden. Bereits in meiner ersten Arbeit habe ich nachgewiesen, daß das Erinnerungsbild der Störungsempfindungen, d. i. die Vorstellung der Empfindung, ihrerseits die gleiche dissoziierende Wirkung auf den Ablauf der (eingeübten, hinreichend unbeur $\beta$ ten) Sprechbewegungen hat wie die erstmalige zufällige Ursache selbst; es tritt an die Stelle einer assoziativen oder primär disso- 
ziativen Ataxie eine sekundür-dissoziative. (Vgl. besonders S. 468 bis 472 meiner zitierten Arbeit). Voraussetzung ist, daß die Vorstellung gefühlsbetont genug ist. Zur Dissoziation kommt es natürlich nicht, wenn die Vorstellung sehr bla $\beta$ ist; in diesem Fall tritt „Vergessen“ ein, wie die Beobachtung zeigt. Wo aber die Vorstellung gefühlsbetont genug ist und die Wiederbelebung der Unlusterregung sich einstellt, da sind die Bedingungen für Sprechataxie als Differenzerscheinung von alt-motorischem Impuls und neu-sensorischer Perzeption (vgl. S. 471 l. c.) unbedingt geschaffen. Ist die Störungsvorstellung abstrakt, d. h. hat sie zum Inhalt die Gefühlserregung und ihre Irradiationen, so ist die resultierende Sprechataxie eine sensorielle Reaktion; hat sie dagegen Berührungs-, Druck- und ähnliche Empfindungen des Tastsinns zum Inhalt, so ist die Bewegungsstörung eine motorische im Sinne der bezüglichen psycho-physiologischen Untersuchung. Also nur in denjenigen Fällen, in denen, klar oder unklar, die Psyche des Sprechenden aus Vorstellungen und Erscheinungen die Reflexion produziert: „Obwohl ich auf diese Stelle des Wortes, auf diesen Buchstaben wieder geachtet habe,

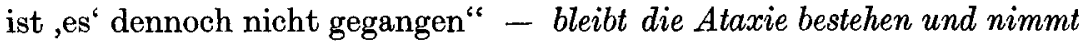
gesetzmäßig bestimmte Formen an. $\mathrm{Zu}$ den assoziativen Bedingungen müssen, wie schon erwähnt, die affektiven hinzutreten, und diese sind bei nervösen und psychopathischen Organisationen vorzugsweise erfüllt; die entsprechenden Bahnungen vollziehen sich bei ihnen außerordentlich schnell. Im einzelnen Falle finden sich die beiden Typen der Ataxien späterhin meist gemischt vor (s. u.).

Für die weitere Darstellung wird mit innerer Notwendigkeit folgende Terminologie durchgeführt werden:

1. Das Wort ,Stottern" fällt als falsch, mißverständlich und entbehrlich fort.

2. Sprachdissoziation bezeichnet primäre Sprechataxie; beide Bezeichnungen können annähernd gleichwertig gebraucht werden.

3. Störungsvorstellung = Sekundärassoziationen der , inneren Sprache".

4. Störungsbewußtsein ist die Organisation von 3.

5. Die systematisierte ,assoziative A phasie" (vulgo „Stottern") besteht aus den jetzt zu beschreibenden Willküren und den höheren Anpassungen, die allgemein der Wiederherstellung der Sprachfunktion dienen.

Der Status des Krankheitsbildes ist jetzt folgender:

1. In der menschlichen Gesellschaft, in einer Summe von Gegenseitigkeitsbeziehungen und -leistungen, bewegt sich ein Individuum, bei dem, unter der Bedingung innererer Aufmerksamkeitserregung, durch Affekt in die Helle des Bewußtseins gestellt, Störungsvorstellungen, mit der Folge sekundärer Sprechataxie, den Sprechanfang im Gebiet der inneren Sprache dissoziieren. Die Störungsvorstellung 
ist komplex geworden, sie hat den Umfang einer Reflexion: „Obwohl ich auf den ..... (Buchstaben) aufpasse, ging es" doch nicht."

2. Der Äußerungstrieb erleidet immer häufigere Schockierungen. Jedes Wiederauftreten der ataktischen Bewegungen bedeutet eine solche für alle Qualitäten bzw. Motive dieses Triebes.

3. Generell ist von Bedeutung, daß das motorisch-dynamische Prinzip der ataktischen Sprechbewegungen sich der beobachtenden Aufmerksamkeit des Sprechenden wie des Zuhörenden aufdrängt.

4. Die „soziale" Seite der Störung tritt immer stärker hervor. Jede Ermahnung, jede Frage ist eine Schockierung vom Werte eines beschämenden Affektes; der dissoziiert Sprechende gewinnt außerdem fast ohne jede Ausnahme die Utberzeugung, daß alle, die sich um seine Störung bekümmern, „es" auch nicht wissen.

Die Häufigkeit des Auftretens der Ataxie ist in diesem Stadium geradezu pathognostisch für den Grad der Affekterregbarkeit und für den Typ der psychopathischen Veranlagung. Bei ganz kleinen Kindern, die erst im Beginn des grammatischen Sprechenlernens stehen, ist alles seelische Geschehen außerordentlich lebhaft; sie nehmen mit größter Begierde die Eindrücke auf, sie machen ihre Versuche, sich in der Umwelt begreifend und beherrschend zurechtzufinden, mit Daransetzung einer oft erstaunlichen Energie, die so leicht durch keine Mißerfolge abzulenken und zu beugen ist. Die Erfahrung lehrt, daß bei frühzeitig zum höheren Begreifen befähigten Kindern gerade das Formen wortfertiger Begriffe einen bezeichnenden Grad dieser Befähigung darstellt. In der Wortsprache begreifen wir erst die Beziehungen der uns umgebenden Menschen zu ihrem Tun und zu uns in einer Art grundsätzlichen Bedeutung. Danach strebt auch das Kind, daß es einen ihm wünschenswerten Grad von Verständnis und von bequemster Einordnung seines Bereichs in den der Erwachsenen erreicht; wenn es ,Fragen und Sagen“" kann, vermindern sich die Reibungsflächen, wächst sein Aktionsbereich. Es ist ja auch für den Erwachsenen angenehm und bequem, wenn er aus Fragen und Antworten ersehen kann, daß das Kind sich in den ihm zugewiesenen Bereich geistig und körperlich eingeordnet hat; beschränken die sozialen Verhältnisse namentlich in den Städten die primitiven Beziehungen der Eltern zu ihren Kindern doch auf sehr oberflächliche und spärliche Gemeinschaften, in denen Fragen und Antworten einen gleichfalls nur geringen Raum einnehmen. Je nach Temperament und Befähigung wird also ein dissoziiert sprechendes Kind früher oder später, mehr oder weniger auffallen, oder seine Selbstbeobachtung und Erfindungsgabe betätigen. Alle Kräftesysteme bewegen sich stets in der Richtung des geringsten Widerstandes (Kassowitz). So ist die Häufigkeit des affektiven oder assoziativen Erreichtwerdens der Störungsvorstellung abhängig von dem Grade der Erregung, die der Verkehr mit den Mit- 
menschen überhaupt verursacht, außerdem von der Erregungshöhe der geistigen Äußerungen und der hauptsächlichen Triebe überhaupt. Beim ataktisch sprechenden Kinde finden sich nun eine Anzahl besonders anhängiger assoziativer Widerhaken. Das ist nach dem Vorstehenden ganz zwanglos begreiflich. Die Höhe der Erregung ist zum einen Teil bedingt durch die hohe Erregbarkeit der Konstitution, des gesamten reaktionsfähigen Zentralsystems, zum anderen Teil durch den von Satz zu Satz sich fortsetzenden Beschämungsaffekt über das ungewollte, der betriebenen Organisierung des Denkens artfremde Mißlingen der Leistung, so daß Antrieb und Hemmung fortwährend wechseln. Dissoziiert sprechende Kinder können in diesem Widerstreit dauernd so stark erregt sein, daß der Anfang eines jeden Haupt- und Tätigkeitswortes von der Störungsvorstellung beherrscht wird. Hier sei wieder im obigen Sinne bestimmt darauf hingewiesen, da $\beta$ das geschilderte Mißlingen ein doppeltes ist: sowohl technisch wie begrifflich; nicht nur der Bewegungsvorgang, als Äußerungstat, mißlingt, auch dem Begriff bleibt gegenüber seiner Wortfertigkeit, seiner logischen Präzision, eine Unschärfe der Bildung wie der Beziehung. Die Grundlage zu dem später noch zu erwähnenden „Esprit d' à peu près" ist geschaffen. - Andere Kinder wiederum sind nur gelegentlich ataktisch; diesen wohnt ein grundsätzlich geringerer Grad der Affekterregbarkeit inne, ihre Vorstellungen und Vorstellungskomplexe sind weniger leicht dissoziierbar. Es hat bei vielen Beobachtungen an ganz Jugendlichen den Anschein, als ob das Wortverständnis und der Wortgebrauch nicht von unten herauf (d. h. eine gut entwickelte und assoziativ reich besetzte Ergopsyche und Stereopsyche werden durch Einordnung des symbolisierten Wortklanges und durch seinen Gebrauch zum sozialen Primitiv-Ich), sondern von oben nach unten stattfindet: ein Primitiv-Ich, das seine lebhaftesten Lustgefühle beim Gebrauch symbolisierter Wortklangbilder und im Vorstellen höherer Begriffe hat, schreitet, durch Fehlleistungen veranlaßt, zur nachträglichen Belebung des Raumanteils und Tätigkeitsbereichs der Dinge und seiner selbst. Diese Beobachtungen würden ja auch dem Wesen der psychopathischen Veranlagung entsprechen. Die Häufigkeit und assoziative Bedingtheit des Bewußt- und Aktivwerdens der primären Störungsvorstellungen ist hiernach an zahlreiche Bedingungen geknüpft, die das konstitutionelle Element ebensowohl wie das eigentliche assoziative erkennen lassen. Darum kommt es zu gerade sprachlichen Dissoziationen bei dieser Gruppe und nicht bzw. nicht ausschließlich zu solchen des übrigen Bewegungsapparates. Übrigens können überall im menschlichen Vorstellungsleben entsprechende Dissoziationen auftreten, wo es sich um Bewegungsvorstellungen und Bewegungsbilder handelt, die in irgendeiner Weise einem $\ddot{A}$ ußerungstrieb als Symbolwerte dienen; die assoziative Bedingtheit ihres 
Auftretens ist der obigen entsprechend (,Schreibkrampf", „Geigerkrampf“, „Platzangst", bei denen entweder die Bewegungsanomalie oder die Psychose überwiegt).

Die Häufigkeit der primären Sprechataxie ist annähernd gleich der Intensität der Erscheinungen. Im späteren Stadium ändert sich das in vielen Fällen.

Die Dauer dieses Stadiums ist sehr verschieden, oft überraschend kurz, bei diesen Wortanfängen anders als bei jenen. Es kommt vor, daß bei Eintritt der hiernach zu beschreibenden Willküren alle primären Ataxien schlagartig verschwinden, es bleibt auch wohl beim gemischten Zustand, auch finden sich in vereinzelten Fällen bei erwachsenen Dissoziierten einzelne primäre Ataxien in reiner Form neben unendlichen Varianten der typischen Willküren oder Sekundärataxien. Man beobachte sprachdissoziierte Kinder nur von Beginn an, und man wird bestätigt finden, daß wir hier von einem klinischen Verlauf sprechen müssen. Früher fing die Klinik der Lungentuberkulose auch etwa bei der Kaverne an.

Es muß durchaus für eine Anzahl von Fällen zugegeben werden, daß die primäre Dissoziation der Anfänge gewisser Worte durch Psychotraumen bedingt sein kann, die als solche aus dem Unterbewußtsein heraus eine latente Affektquelle bleiben und in dieser Weise eine Art Fremdkörperdasein zu führen fortfahren können. Es hieße aber den Tatsachen Gewalt antun, wenn man leugnen, verkennen oder übersehen wollte, daß die sekundäre Sprechataxie (die Willkürentwicklung, s. u.) auch diese Fälle zu den sprachlich Dissoziierten gesellt, und nicht einfach zur Gruppe der Angstneurotiker aus traumatischer Ursache. Es ist auch praktisch viel wichtiger, dem Sprachdissoziierten das Perverse seiner Sekundärataxien zu Bewußtsein zu bringen als womöglich nach erotischen Psychotraumen zu analysieren. Selbstverständlich werden auch eine Anzahl Angstneurotiker sprachliche Dissoziationen gehabt haben können; wenn sie sie später nicht mehr haben, so sind sie eben frei auch von den Sekundärataxien, denn diese bedingen erst den charakteristischen Dauerzustand der schweren neurotischen assoziativen Ataxie. Eine Angstneurose auf der Basis einer der psychoanalytisch bekannten Schockierungen kann sich vor, neben und nach einer assoziativ oder dissoziativ bedingten Aphasie vorfinden; die letztere wird aber erst bei ihrem späteren assoziativen Ausbau eine wirkliche Komplexgemeinschaft hervorbringen. Ich habe unter den wenigen mir bekannt gewordenen Fällen analytisch die Grenze nur sehr schwer ziehen können; jedenfalls genügt für die Sprachbeherrschung durchaus nicht eine Lösung der rein affektiven Komponente. Man kann auch beobachten, daß die primäre Ataxie sich von einem Haupt- oder Tätigkeitswort zum anderen fortpflanzt. Leider werden die für gerade diese Beobachtungen geeig- 
netsten Objekte, die hereditär psychopathischen und gleichzeitig im Beginn der Dissoziation stehenden Kinder, nicht frühzeitig genug zum Facharzt gebracht. Es wäre wertvoll, festzustellen, wie und in welcher Zeit die Erwerbung dieses oder jenes Begriffswortes usw. stattfindet, in welcher Reihenfolge sich die Elemente der Primitivpsyche entwickeln, und an welchen Worten die primäre Dissoziation zuerst auftritt.

Man kann sich durch Nachahmung der primär ataktischen Bewegungen leicht davon überzeugen, daß die Störungsempfindungen, besonders die des Tast- und Bewegungssinns, recht lebhaft sind und nach ganz kurzer Frist scharf vorgestellt werden können.

\section{V.}

Dem Sprechakte, d. i. dem Aktivwerden des Äußerungstriebes, der zugleich die Gefühle des körperlichen wie des geistigen Sich-Anstrengens enthält, bieten sich von jetzt an Widerstände dar in Gestalt von neu assoziierten Berïhrungs- und Spannungsempfindungen usw., auch von Wiederholungen im Bewegungsvorgang und von abwegigen Vorgängen der inneren Sprache; die expressive Seite der Psyche findet im Zeitminimum assoziierte Elemente in ihrem Aktivitätsbereich vor, die mit zur Funktion gelangen und affektiv oder reflektiv erregend wirken bzw. gewirkt haben. Der Sprechvorgang fließt durch einen erweiterten Assoziationsmechanismus. Die motorische Ausnahmeleistung wird, als Fehlleistung gewertet, naiv (d. h. nach dem Prinzip der Erhöhung der dynamisch-motorischen Willkürleistung), die sensorische, d. h. die assoziative, Ausnahmeleistung wird nach dem assoziativen Prinzip (Prinzip der Neurose) wiederherzustellen gesucht. Dabei finden typische Entwicklungen statt, die als „Klinik" der betr. Prinzipien zu werten sind ${ }^{1}$ ).

Was man theoretisch annehmen muß, geschieht nun auch in der Wirklichkeit. Das sprechende Individuum schämt sich seiner Ataxien, es ärgert sich über sie, es erschrickt vor ihnen, es empfindet Unlust im höchsten Grade und gerät aus diesen Gefühlsalterationen über kurz oder lang in einen Zustand von Gereiztheit und Erregung vor dem

1) Die nachfolgend aufgeführte schematische Darstellung der Entwicklung der Gruppen der assoziativen Sprachstörung stammt von mir. Ich habe sie zuerst in einem Vortrage gegeben, den ich vor der Medizinischen Gesellschaft in Marburg im Winter 1913 hielt, sodann in einer der hier festgelegten Form fast genau ent. sprechenden in der Diskussion zu einem Vortrage von Fröschels im Escherich. Pavillon der Medizinischen Klinik in Wien vor den Kinderärzten Wiens im Januar 1914. (Inzwischen bin ich durch meine Kriegsteilnahme genötigt gewesen, mein Gebiet andern zu überlassen, denen es die äußere Lage ermöglichte, diesen Dingen obzuliegen und die neueste Literatur zu berücksichtigen.) Diese Arbeit ist zugleich ein Dank an die Medizinische Fakultät Marburg für Verleihung eines Stipendiums aus der Bosestiftung, besonders an dis Herren Mannkopff $(\dagger)$, Tuczek und MatthesKönigsberg. 
Sprechen, dem sich, wenn auch nicht bei allen Fällen, sehr frühzeitig ein echtes Angstgefühl beimischen kann. Damit ist die Willkür. anwendung ganz von selbst aufgerufen, die nach einer Wiederherstellung des normalwertigen, sozial reibungslos brauchbaren Klangwertes der Sprache mit der Selbstverständlichkeit des Sprachäußerungstriebes streben muß.

Die Begrenzungen, die jede Schematisierung erfordert, sind so zu verstehen, da $B$ die Utbergangsformen nicht berücksichtigt sind; überhaupt betrifft die Schematisierung beim selben Individuum mehr das Schicksal einzelner Laute und Dissoziationen als zugleich das Gesamtbild der assoziativen Störung. So können beim selben Falle Dissoziationen aus allen drei Gruppen vorhanden sein. Auch soll noch besonders abgehandelt werden, in welchen Fällen die assoziative Aphasie sich im Anschluß an primäre Sprachdissoziation auszubilden pflegt. Das Schema betrifft als solches also alle in der Praxis allgemein bekannten Fälle von ,Stottern“"

I. Motorisch-dynamisehe Form: erstes Stadium bzw. erste Form der assoziativen Rekonstruktion. Durch Verlassen der normalen auditivsensorischen Sprachkontrolle und Einführung der, durch die primären (primitiven) Störungsvorstellungen herbeigeführten taktil-motorischen Kontrolle wird der Sprachäußerungswille dynamisch motiviert; d. h. er produziert, gemäß der Vorstellung des Widerstandes bzw. der Vorstellung des motorischen Mißlingens ein subjektives Übermaß von motorisch-dynamischer Willkür, als deren Ergebnis grobe „Dynamisierung“" der Sprechbewegungen, und zwar der Artikulationsstelle, resultiert.

Also Status: 1. Sprachäußerung: assoziative Dynamisierung.

2. Mitarbeit von Hilfsmuskelgruppen.

3. Irritations- und Irradiationsbewegungen entfernterer Muskelgruppen, durch Irradiation der psychischen Erregung bis in das autonome Nervensystem entstanden.

4. Desgleichen als „Ablenkung“ (Übergang zu II), später willkürliche Benutzung von 2 und 3.

II. Sensorisch-aphatische Form: Zweites Stadium bzw. zweite Form der assoziativen Rekonstruktion: Der "inneren Sprache" ist durch Urteilsbildung der Begriff „,es geht nicht" assoziiert; der Äußerungswille wird verbalsensorisch motiviert. Dazu treten, frühzeitig oder zur Zeit der Pubertät, ethisch-ästhetische Hemmungen in zunehmender Stärke: allerlei Praktiken der Dissimulation werden produziert, gegen die Vorstellungen und Funktionen der ersten Form wird mächtige Verdrängungsarbeit geleistet.

Also Status: 1. Seltenes Auftreten der dynamischen Motivierung.

2. Die dynamischen Willküren werden vor dem Urteil zu subjektiven Unmöglichkeiten. 
3. Umgehung derjenigen Worte, deren Hervorbringung man nicht im dynamisch verdorbenen Zustande haben will.

4. Entsprechend dieser negativen Willenstendenz ausgiebigste Produktion von Ähnlichkeitswerten (,Verschiefung“ der Logik), Paragrammatie, wie bei organischer Aphasie.

5. Gesteigerte Benutzung von Mimik und Geste, teils zur Ablenkung (Kräfteverbrauch, Ermüdung, Verlegung der Aufmerksamkeit des Zuhörers), teils zur Unterstützung der Glaubhaftmachung der gespielten Nachdenklichkeit: Rekonstruktion der Sprache vor dem Ich, also Übergang zu III.

III. Moralisch - psychopathische Form: Drittes Stadium bzw. dritte Form der assoziativen Rekonstruktion: unter Anerkennung des das Sprachvermögen betreffenden Urteils: ,es geht nicht“ äußert sich die Störung des Selbstbewußtseins (hervorgebracht durch Funktionsunsicherheit, Urteilsumwertung und dauernde Hilflosigkeit gegenüber der assoziativen und sozialen Impotenz) in der Produktion des Begriffs „Ich kann nicht"“. Der ÄuBerungswille wird moralisch motiviert; entsprechend diesem Urteil wird eine Umwertung aller Beziehungen des sprechenden Individuums zu seiner Mitwelt vorgenommen. Das ,Ich“ ist zurüekgewonnen durch perverse Bewertung des Störungs-Ich und wird schließlich völlig ersetzt durch dieses. Vorherrschen der negativen Gefühlstöne und -erregungen im ganzen produktiven bzw. geäußerten oder äußerungsbereiten Seelenleben. Ausbildung noch anderer ,Ich kann nicht". Die klinischen Bilder der Imbezillität, der paranoiden, debilen usw. Form der Psychopathie und der Hysterie treten hervor, dargestellt und ausgefüllt mit den Inhalten von I, II und III auf der Grundlage der Vorstellung sprachlichen Unvermögens. Der ,Geltungswille" produziert Bizarrerien.

Also Status: 1. Sprachlich sind kaum wesentliche Sekundärataxien festzustellen; oft müssen sie zum Zweck der Exploration erst künstlich aktiviert werden (Vorsicht!).

2. Gelegentlich ist II $5 \mathrm{zu}$ bemerken.

3. Enorme Produktion von umgewerteten Urteilen verdeckt fast ganz die Vorstellung sprachlichen Unvermögens, bis zur sozialen und ethischen Minderwertigkeit und Umsturzwertigkeit der Person.

4. Starke Affekte, Angstzustände, Spannungszustände, abnorme psychische Reaktionen (Fugues, Pseudologie, Zwangshandlungen usw.).

5. Mitaktivierung anderer psychischer Anomalien durch die chronische Affektivität, Hervortreten falscher und echter Begabungen, Übergänge zu hysterischen Zustandsbildern durch „Surrogat"-Leistungen ${ }^{1}$ ).

1) Vgl. meine Arbeit: Uber die Disposition der Stotterer-Psyche zu asozialer Entwicklung. Arch. f. Krim.-Anthropol. u. Kriminalistik 49, 149_174. 1912. 
Um Irrtümern vorzubeugen sei nochmals erwähnt, daß unter ,Sekundärataxien" diejenigen der ersten Form verstanden werden. (Eigentlich sind es sensu strictiori die tertiären Bildungen; denn die Ataxien, die z. B. durch die erste Einwirkung einer Causa nocens, oder bei assoziativer Sprachentwicklung entstehen, sind die eigentlich primären. Die eigentlich sekundären aber sind die, die durch die bewußte, quasi experimentelle Spaltung des Sprechvorgangs in einen sensorischen und einen motorischen Anteil (vgl. meine zit. Arbeit S. 471) bzw. in einen neuen und einen alten Anteil bedingt werden; die hiernach einsetzende Dynamisierung brächte logischerweise dann also eine dritte Art von Störungen hervor. Es empfiehlt sich jedoch für die klinische Bezeichnungsweise, die aphatischen Störungen der I. Form als Sekundärataxien zu bezeichnen, weil die ,eigentlich“ primären und ,eigentlich“ sekundären Dissoziationen funktionell gleichwertig sind und zeitlich wie funktionell vor den Erscheinungen des ausgebildeten Zustandes liegen, der allein den pathologischen Wert einer Krankheit hat.) Die Bezeichnung ,Sekundärataxie" wird also gleich,,dynamisiertes Artikulationsmoment" festgelegt.

Die häufigsten Bilder sind die assoziativ bedingten der dynamischmotorischen Sprachstockung. Nicht alle Primärdissoziationen gehen von I über II zu III über; das ist je nach der Festigkeit des assoziativen Sprachgefüges, je nach der gesamten Persönlichkeit und je nach dem Fehlen oder Hinzutreten weiterer schädigender Ereignisse individuell und graduell verschieden. Auch das primär-ataktisch sprechende Kind wird nach und nach, nicht immer sofort, von seinen Ataxien moralisch betroffen, die Beeinträchtigung des Selbstbewußtseins und die Motivierung der Aufmerksamkeit haben dem assoziativen Sprachgefüge gegenüber verschieden schwere oder leichte Arbeit, es zu lockern. Die vitiose Assoziation der Störungsempfindungen führt selbst bei organischer Ursache der Primärataxie (z. B. nach Laesio medullae oder cerebri) nicht in allen Fällen zur dynamischen Motivierung des Sprechwillens bzw. bleibt die Apperzeption derselben aus. Die Psychoanalyse deckt nicht selten bei selbst ausgesprochenen Zuständen auf, daß in der Kindheit primäre oder selbst sekundäre Ataxien bei Worten bestanden haben, die im gegenwärtigen $\mathrm{Zu}$ stande, auch affektiv, ohne Dissoziation und deren Folgen gebraucht werden. Die Ursache liegt darin, daß die betr. geistige Organisation nicht fähig war, die entstandenen Empfindungen durch geeignete Perzeption zu Vorstellungen zu erheben, sei es, daß der ursächliche Zustand nicht lange genug anhielt, sei es, daß ein pathischer Zustand der Psyche aus anderen Ursachen ausblieb. Von den eigentlichen organischen Ursachen, die in der molekularen Reaktivität der leitenden Bahnen, überhaupt der anatomischen Grundlagen des Denkens liegen, ist an anderer Stelle gehandelt ${ }^{\mathbf{1}}$ ).

1) Hiermit ist, wie ich gegen die Neurologie bemerken muB, die Abgrenzung gegen Sprachataxje nach organischer Läsion noch nicht erschöpft. 
Nicht nur die bisherigen theoretischen Begründungen, sondern auch zahlreiche Selbstzeugnisse belegen die Tatsache, da $B$ die I. Form der assoziativen Sprachstörung eine Willkür ist. Die auditiv-sensorisch, beim normalen Menschen unter indifferenter Aufmerksamkeitseinstellung von Anfang der fertigen Wortsprache an bis zum Tode nur und ausschließlich auditiv-sensorisch regulierten Sprechbewegungen bringen Lautgebilde hervor, die es eben in Anbetracht der oben (Kap. IV, Anfang) geschilderten übrigen Assoziationen nicht vertragen, daß einzelne taktil gekennzeichnete Momente und Bewegungsgruppen dynamisiert werden. Jeder kann das nachahmen. Nur die psychopathische Psyche gibt den genügenden Widerhall für die Erinnerungsbilder der Primärerscheinungen. Wer dauernd durch Nachahmung dissoziiert bleibt, ist eben dem unentrinnbaren Trugschluß der sekundärataktischen Periode genau so gut wie der das Beispiel Gebende zum Opfer gefallen und bleibt genau ebenso sekundärataktisch aus lediglich assoziativer Ursache, wenn die wiederholte Schockierung der Psyche einen ausreichenden $\mathrm{Zu}$ stand von Pathos bewirkt hat. Gerade die Fälle, in denen kleine Schulklassenendemien von assoziativen Sprachstörungen vorkommen, beweisen klipp und klar den rein psychischen Mechanismus. In derselben Familie kann ein Kind in der üblichen Weise erkranken, mehrere andere durch Nachahmung, und vielleicht ist das aus wirklich krankhafter Ursache dissoziierte Kind in den ersten Schuljahren oder nach Ablauf derselben oder nach einem einzigen Heilkurs lebenslang von seiner Störung befreit, während die Nachahmer lebenslang darin verharren trotz zahlreicher Kuren. Übrigens ist die Situation einer Schulklasse dem Entstehen einer „Einstellungspsychose“ überaus günstig; sie kann Seelenzustände liefern, die außer für die Entstehung des Symptoms zugleich für tiefere „Psychogenien" vorzüglich geeignet sind.

Die Dynamisierung der assoziativ bezeichneten Momente im Bewegungsablauf ist grundsätzlich von doppelter Wirkung: einmal „hilft“ es im naiven Sinne, denn die bekannten lächerlichen und schleppenden Lautwiederholungen machen einer kürzeren, tragischeren und neuartigen Bildung Platz. Es dauert oft geraume Zeit, bis dem Sprechenden überhaupt zu Bewußtsein kommt, daß ein neues Störungsprinzip an Stelle eines alten getreten ist; in den meisten Fällen, d. h. in denen die erste (I.) Form als Haupttyp lebenslang bestehen bleibt, ist das kritische Bewußtsein dauernd unfähig, diesen Vorstellungskomplex, so leicht zu lösen er auch ist, wieder abzubauen. Die Dynamisierung der assoziativ bezeichneten Momente bedeutet aber auch für die Stärke des Äußerungstriebes eine Subtraktion; erschöpft man sich in Bewegungswillkür, so vermindert man entsprechend die Geneigtheit zu sprachlicher Mitteilung, oft so sehr, daß einfach aus dem Zustand des Sprechenwollens in den des willkürlichen, alle sozialen Folgen auf sich nehmenden Sprach- 
verzichtes übergegangen wird. Zwischen diesen beiden Extremen: der lebendigen wirksamen Sprechtat und dem gewissermaßen „Verschwinden" des Sprechenden (in seiner doppelten Eigenschaft als Sprechender und als soziales Individuum) gibt es alle Übergänge; es kommt hier auf den Hinweis an, daß die Umformung von ursprünglich psychischer Energie in dynamische neben einer qualitativen auch eine quantitative Änderung des Äußerungstriebes bedeutet, die wir in allen weiteren Entwickelungsformen wiederfinden werden. Ohne diese den psychischen Kräftehaushalt betreffenden Bemerkung ist der Zustand und die Entwicklung aller nur vorkommenden assoziativen Seelenstörungen vom motorischen Symptom bis zur Geltungsumwertung des Sozialitätswertes gar nicht zu verstehen.

Die dynamischen Bildungen wurden als kürzer, tragischer und neuartig bezeichnet. Der Sprechende hat von ihnen einen Gewinn, denn, was die Ähnlichkeit dieser Produkte mit dem vorgestellten, innerlich gesprochenen Idealworte betrifft, so ist diese wirklich eine größere Annäherung als die Form der lautwiederholenden primären Ataxie. Dafür aber drängt sich der Urteilskraft das Bewußtsein des Zeitverbrauchs während der (scheinbar) unberechenbar langen Dauerkontraktionen der enorm verlängerten Artikulationsmomente vorstellungs- und gefühlsmäßig stark und stärker auf; eine Reihe von nah-oder fernliegenden Reflexionen findet währenddessen statt. Man kann beobachten, daß der Sprechende, während er seine ganze örtliche dynamische Energie der Artikulationsstelle z. B. des Lautes „K“ zuwendet, sich im Zimmer umsieht, einen Knopf schließt oder ein paar Zeilen mit den Augen weiterliest. Subjektiv wie objektiv zählt die reine Dauerkontraktion nicht als Sprache bzw. als Sprechen; man kann sich davon überzeugen, wenn man mit geschlossenen Augen einem Sekundärataktischen der I. Form zuhört und sich auf die wirklich hervorgebrachten Wortklänge konzentriert und von den vereinzelten ,,vorbeigehenden “ Explosionen der harten Laute absieht, die mitunter noch Ähnlichkeit mit der alliterierenden Form der Primärataxie haben können. Für den Sprechenden ist das betreffende Wort erst dann ausgesprochen, wenn annähernd normale Kraftanwendung und desgleichen Lautierung vom Bewußtsein registriert wird; der Maßstab für normalwertige Wortklänge geht nie verloren, lebenslang begnügt sich der assoziativ A pathische nicht mit Mißbildungen, sondern strebt stets nach einem Gebilde, das der inneren Sprache konform ist hinsichtlich seiner auditiven wie seiner taktilen Werte. Er denkt überhaupt genau so, wie jeder andere Mensch unter der gleichen irrigen Voraussetzung denken würde. Ganze Lehrgebäude von Ärzten und Laien sind auf der Voraussetzung aufgebaut, der Sekundärataktische könne infolge wirklicher organischer Ursache etwas nicht; freilich ist richtig, daß der Ataktische mit den Mitteln, mit denen er es versucht, 
nämlich mittels der Dynamisierung der Artikulationsstellen, wirklich keinen normalen Bewegungsablauf zustande bringt, und auch das teilt er mit nicht weniger wie allen seinen Mitmenschen. Diese Forscher haben, irrtümlich oder aus unzureichender Begründung, nachzuweisen unter. lassen, warum und wie die bewußte Dynamisierung der Artikulationsstellen (jeder Sekundärataktische wird auf Befragen antworten, daß er das tue in der Absicht, richtig zu sprechen, und wird mit seinen Willküren aufhören, wenn er den Willen zu sprechen aufgibt) einer pathologischen Erscheinung aus der Welt der grob organisch lädierten Materie, etwa einem Krampf gleichzusetzen sei. (Ich wies übrigens in früheren Arbeiten schon darauf hin, daß die Dynamisierung stets in der Richtung des Weitersprechens stattfindet, nicht aber umgekehrt in der Art besteht, daß sie geeignet wäre, eine Gegenleistung gegen einen hypothetischen Spasmus darzustellen.)

In unheilvoller Weise kommt zu jedem die Fehlleistung begleitenden unmittelbaren Unlustgefühl die Erkenntnis, daß man, ein Bild verzerrter, gequälter und fruchtloser Anstrengung, seine Stellung zu seinen Mitmenschen durch Erregung von Mitleid verbessere. In nicht allzu langer Zeit fällt die wahrhaftige innere Ehrlichkeit dieser Geltungsänderung zum Opfer. Kinder wie auch Erwachsene werden bald ,schwer erziehbar, moralisch undurchsichtig, zu starken Affekten geneigt, häufig verlogen, aller Verstellungen fähig“". Alle Erfahrung und alle Kenntnis vom Wesen psychopathischer Zustände spricht dafür, daß man zu wesentlichen Graden dieser abnormen Veranlagung schon vorgebildet sein muß, um sich so leicht und so stark auf ein abnormes Verhältnis zur Umgebung einzustellen. Daraus erklärt sich auch die allbekannte Bereitwilligkeit sekundärataktischer Kinder, zu Nutz und Frommen ihrer Bequemlichkeit, Unverantwortlichkeit und Unansprechbarkeit ihre Ataxien rein willkürlich hervorzubringen, d. h. ohne assoziativ zwangsläufiges Einspringen der Unfähigkeits- bzw. Störungsvorstellungen, lediglich mit der Vorstellung von der Unmöglichkeit, mit den gewählten Mitteln der dynamischen Leistungsvergröberung die Sprechleistung in den Rahmen der sozial bzw. äußerlich zur Verfügung stehenden Zeit hineinzupassen. Das ist sehr leicht zu erlernen, und jeder einigermaßen ehrliche Sekundärataktische wird dies, womöglich nicht nur für die Schulzeit, offen eingestehen können.

Diese Verhältnisse werden durch die weiter unten aufgeführten Ausfallserscheinungen noch ergänzt werden.

Die Mitarbeit der Sprach- und Atmungs-Hilfsmuskeln ist als Erfindung und Bedarf an Entlastungsgebiet und Entlastungsarbeit aufzufassen. Die Einbeziehung von Bewegungen der durch Affekterregung anfangs unwillkürlich innervierten Muskelgruppen in den Sprechakt vollzieht sich entsprechend. Dem kommt entgegen, daß ganz allgemein 
bei stark fühlenden Menschen (z. B. auch bei den Romanen) die Mimik als ausdruckergänzend empfunden wird. Auch hier ist von grundlegender Bedeutung für die weitere Entwicklung, daß diejenige dynamische Energie, die für die Innervation fernliegender Muskeln (aber dem Sprechakte assoziiert!) verwendet wird, tatsächlich der an der Artikulationsstelle (als der Stelle der taktilen Maximalempfindung) angesetzten Energie abgezogen wird, so daß eine Beschleunigung des "Loskommens" dabei herauskommt. Daher wird sehr häufig mit Fußstampfen, Schütteln usw. gearbeitet. Alle diese Bewegungen hören auf, wenn der Wille zu sprechen dem sie assoziiert sind, aufgegeben wird, ohne da $\$$ die mindeste andersartige Bewegungsalteration der zur Hilfe gezogenen Muskeln für ihren ursprünglichen Funktionsbereich vorhanden ist ${ }^{1}$ ). Auch Ties jeder Art werden eingeübt; sie dienen zum überwiegend größeren Teil in dem hier angegebenen Sinne der dynamischen Entlastung der Sprachwillkür, werden aber auch zum Teil aus psychologischen Gründen erfunden und illustrieren so die erwachende Neigung zum Anders-Sein, das Gefallen am Krankhaft-Auffälligen. Auch Tics fernliegender Muskeln kommen zur Beobachtung (Fuß usw.).

Wenn auch, namentlich bei unbequemer Belastung der Verantwortlichkeit, überhaupt bei Ansprüchen an die aktive Leistung und Anpassung des Kindes, der Schaden leichtfertig und absichtlich in den Kauf genommen wird, der durch Sprachverweigerung in der Form willkürlichen Hervorbringens der dynamischen Ataxien im kleinen Sozialitätsbereich entsteht, so befindet sich doch das Individuum in einem Zustande von Pathos, den es als krankhaft zu empfinden nie aufhört. „,Die ich rief, die Geister, werde ich nicht mehr los." Es geht aber nichts über die gesunde Rücksichtslosigkeit der normalsprechenden Mitmenschen. Der Assoziativ-Aphatische bekommt die Konsequenzen seiner abnormen Sprachführung, seiner undurchsichtigen Selbstbeurteilung seines Leidens (das er bald als Krankheit und soziales Scheusal, bald als unantastbares „Tabu“-Heiligtum behandelt), seine mangelnde Gegenwehr gegen das verdorbene Sprechen gründlich zu fühlen. $E s$ ist auch das unbestreitbare Recht der menschlichen Gesellschaft, daß sie sich instinktiv dagegen wehrt, ein Refugium für unbegreifliche Schwächlinge zu sein. Die Sozialität, eine Arbeitsauslese der Tüchtigen, wird immer eine sehr große Maschine sein, innerhalb deren die Grundbedingung des Dabeiseins ein Reibungsminimum im Muß-Ablauf bleibt,

1) Der Begriff der im psychischen Ganzen eingeordneten „begrifflichen“ Bewegung sollte in der Muskelphysiologie so eingebürgert sein, daß eine Verwechselung mit Jackson scher Rindenerkrankung eigentlich unmöglich sein sollte bei Untersuchern, die über Aphasie schreiben. Die Stellung dieser assoziativ. aphatischen Vorgänge zum Gebiet des Pallidum-Syndroms und der striären Bcwegungsstörungen bleibt späterer Bearbeitung vorbehalten. 
der sich zwar auf verschiedene Weise, aber am wenigsten durch moralisch begründete Tempoverzögerungsabsichten beeinflussen läßt. Das Leben verlangt eben überall mehr anpassungsgehöhten Instinkt und Trieb als subjektive Einzel-Assoziation und Vorstellung. Aber es liegt hier eine, wenn auch ungleich große Schuld auf beiden Seiten. Den innerlich oder chirurgisch Kranken weist der eigene und der Laieninstinkt zum Arzte. Nicht so den Sprachgebrechlichen. Die Erfahrung, daß die Mehrzahl der Assoziativ-Aphatischen nur mehr oder weniger gebessert durchs Leben gehen trotz aller schlechten und guten Kuren, ist so bekannt, und das Mißtrauen in den ehrlichen Willen der Leidenden, sich Mühe zu geben, um den gehabten Nutzen zu erhalten, gründet sich bei „Freund und Feind" in so hohem Maße auf die auch in anderweitigen Lebensbetätigungen bewiesene Charakter- und Willensschwäche, daß man sie zu vernachlässigen instinktiv geneigt bleibt. Wenn man sich jedoch vergegenwärtigt, daß im Beginn des Leidens in den weitaus meisten Fällen relativ leicht geholfen werden kann, so ist das doch nicht als richtig zu bezeichnen. So schleift sich das Zustandsbild immer mehr ein, bei der menschlichen Gesellschaft wie beim Sekundärataktischen. Immer wieder rennt die Phantasie gegen die unerklärten Erscheinungen an und eilt der Situation voraus, im voraus die Sätze sich innerlich durchsprechend und durchprüfend, wie dieses oder jenes ,,schwere“ Wort durch eins von gleicher oder ähnlicher Bedeutung zu ersetzen ist; dabei steigert sich die Angst im Quadrat der Annäherung an den Schauplatz der Rede. Am Ziele bricht das mühsam gefügte Kartenhaus des Ausgedachten elend zusammen, ein arges Kauderwelsch von schiefdeutigen und dazu noch immer zahlreicher zuströmenden Worten wird mühsam herausgehaspelt, mit verzweifelter Ergebung in die widerwärtige Rolle wird nach der dynamischen Methode darauf losgearbeitet, nur um ,fertig" zu werden; und die Mitmenschen stehen ergeben und unwillig, innerlich lachend oder innerlich geärgert, je nach Temperament und Situation, dabei und wissen in aller Welt nicht, ob überhaupt, und wie sie helfen sollen.

Wenn man annehmen wollte, daß dieser Zustand so bleiben könnte, so hieße das die Natur eines Triebes verkennen. Selbst in den Fällen, in denen in der wirklich geäußerten Sprache die dynamischen Willküren das Bild beherrschen, bildet sich der letztgeschilderte Seelenzustand aus, und das ungeheuere Utbergewicht der inneren Sprache vor jeder anderen Art von Vorstellungsleben kennzeichnen ihn vor anderen Formen der Psychopathien mit und ohne sekundärassoziative „Pfropfgebilde“. Trotz aller Angst und trotz aller technischen und logischen Selbsthilfsversuche bleibt die Sprachäußerung dieser Gruppe stets durchsetzt von den so charakteristischen dynamischen Willküren. 
Die zweite große Gruppe, die sensorisch-aphatische Form, ist mit kurzen Worten charakterisiert als die Eroberung des sprachlichen Denkens von der Unfähigkeitsvorstellung auf dem Wege der Reflexion, und, in geringerem Maße, durch Affektwirkung. Von den dynamischen Willküren hat sich der Sprechende abstrahiert; die oft fast maximalen Kontraktionen, die brutalen Vergewaltigungen der Artikulationsstellen haben gröbste Empfindungen von sprachfremdem Tun zu Bewußtsein gebracht, und die Vorstellungen davon haben, bei Gegenüberstellung mit dem gesunden Sprachablauf, ein Werturteil - nicht etwa über die angewendete Methode der Dynamisierung, sondern über ihr praktisch hervorgebrachtes Endglied, die Lautzerquetschung, gebildet. Dies lautet: „Es geht nicht.“ In diesem Urteil ist die ganze einseitige Schiefheit der in der Anlage bereits vollendeten, einer Spaltung der Persönlich keit schon gleichkommenden Störung des Selbstbewußtseins und der ganzen Affektlage zu erkennen $\left.{ }^{1}\right)$. Gemä $B$ der Kürze der Zeit, in der sich die pri. mären Störungsvorstellungen assoziierten, ist die Erfindung der bewußten (sekundären) Willküranwendung zum Zweck der motorischen Rekonstruktion des Sprachbildes schneller oder langsamer ins Unterbewußtsein gedrängt oder gesunken. Bewußt ist nur, was eben in dem genannten Werturteil sich verkörpert: die Unfähigkeit, das richtige, innerlich vorgestellte Sprachbild hemmungslos und ohne Zeitverlust zu bekommen. Dies Urteil ,es geht nicht" ist beachtenswert in bezug auf seine Bestandteile; da es nur dadurch sich behaupten kann, daß es die Tatsache des Normalsprechens (das ohne Einschaltung der Störungsvorstellung bei jedem Assoziativ-Aphatischen grundsätzlich lebenslang besteht und bestehen bleibt) nicht in sich einbezieht, so ist für sein Zustandekommen, außer der Fehlleistung und der falsch motivierten Abstraktion von den Tatsachen, noch ein Teil Suggestion, sowie Affekt-

1) Manche Untersucher scheinen Schwierigkeiten zu haben, in kurzer Zeit bei assoziativ Aphatischen des motorisch-dynamischen und des sensorisch-aphati. schen Typs diese stets vorhandene Fähigkeit zu vollkommen physiologischem Sprechen demonstrandi oder curandi et suggestionis eausa zu erzeugen; das ist aber gar nicht schwer, ich persönlich erziele es bei genügend ansprechbarem Verstande (Lebensalter oberhalb zehn Jahren) fast ausnahmslos innerhalb der ersten zwei Stunden. Ebenso mißlingt manchen Untersuchern die Vorführung der dynamischen Symptome bei der zweiten Gruppe gerade in foro, $d . h$. vor dem Kolleg usw. Man muß nur die erforderliche Affektlage und entsprechende Einstellung der Aufmerksamkeit psychologisch herbeizuführen verstehen. Freilich können hier Fehlreaktionen - also Normalsprechen - eintreten, wenn der Demonstrandus zu denen gehört, die in foro durch Induktion in eine stark positive Gefühlslage versetzt werden; aber man lasse sich nicht die Mühe verdrießen, lange genug zu explorieren, man findet dann zweifellos in der Mehrzahl der Fälle das Hervortreten von Phantasiewerten, eingebildeten Tugenden, Pseudologie, phantastische Produktionen jeder Art, die absolut charakteristisch für die Zagehörigkeit zur sensorisch-aphathischen oder abstraktiv-psychopathischen Gruppe sind.

z. f. d. g. Neur. u. Psych. LXXIX. 
verlagerung und grundsätzliche Neigung zur Bildung überwertiger oder zwangsläufiger Vorstellungen anzunehmen, daneben aber auch eine überhaupt für den ganzen Krankheitszustand charakteristische Vormachtstellung des sprachlichen Vorstellens vor dem Raum- und sonstigen realen Vorstellungsbesitz. Immerhin bleibt das Selbstbewußtsein der ganzen Persönlichkeit noch so weit erhalten, daß das Ich sich seiner Unlust und Fehlleistung in der Weise entäußert, daß es seine gesamte Sprachfunktion als motorische Fehlleistung wertet, betrachtet und abstößt, indem es sich sensorisch zu ihr einstellt (Prinzip des Erwartens eines aus der Empfindungssphäre stammenden "Signals" unter dem teils propulsiven, teils prohibitiven Antrieb der Angst) und eine gewaltige Verdrängungsarbeit gegen die dynamischen Willküren leistet.

Die Sprachäußerung steht bei dieser Gruppe, wie schon gesagt, unter dem Zeichen der sensorischen Rekonstruktion. Die Erregung führt die Vorstellung, daß dieser oder jener Wort- oder Silbenanfang ,nicht geht", assoziativ ins Bewußtsein. Gerade die im fortgeschrittenenen Lebensalter erwachten ethischen und ästhetischen Gefühle schwellen mit ganzer heller Bewußtheit und höchster affektiver Stärke den Vorstellungen der inneren Sprache zu; der Entbehrungsaffekt, die Erregung darüber, daß das ersehnte normale Sprachklangbild ,,jetzt gleich" erscheinen soll, wird größer als die ungeheure Unlust, das dynamisch verzerrte „Es geht nicht" gebrauchen zu müssen, und - da kommt ein Stück Satz hervorgeschossen, oft wie die Kartoffeln aus dem Sack, aber trotz aller Hast, aller Angst und allen Erstaunens kommt die Erleichterung obenauf, da $\beta$ man doch seinen Gedanken in der innerlich vorgestellten Form herausgebracht hat. Immer geläufiger wird dieser Mechanismus. Die inneren, mächtige Gefühlsspannungen darstellenden Erregungen werden bald maskiert durch Geste und Mimik des Nachdenklichen, Vergeßlichen, Zerstreuten, Aktiv-Intelligenten, Heiteren, Zornmütigen usw.; sie werden auch bald direkt als Spracherleichterungsmittel in diesem Sinne benutzt: man denkt intensiv an etwas anderes so lange und so intensiv, bis das zur Aussprache bestimmte Stück sprachlichen Vorstellens so gefühlsarm und vorstellungsbla $\beta$ geworden ist, daß keine Erhöhung der den Äußerungstrieb begleitenden ängstlichen Erregung mehr riskiert zu werden scheint. Wehe, wenn das Kunstwerk, als welches dies Kunstmittel zu bewerten ist, etwa so intensiv angewendet wurde, daß sich der grammatikalische Zusammenhang des Satzes im Dunkel verlor! Mit der wieder neu motivierten Aufmerksamkeit stürzt die Horde der gefürchteten Störungsvorstellungen sich wiederum auf die verschüchterten und auf möglichst geheimer Ausreise in die böse Welt begriffenen Gedanken, und die ,gute Form nach außen" ist, elend in Blamage und Ungeltung verwandelt. 
Gemäß dem Vorherrschen der Tendenz, das Bild des Ausgesprochenen möglichst natürlich zu machen, werden eine Anzahl von „Hilfen“" ausgebildet, die zum Teil sozial recht unartig sind. Die motorischen Vergewaltigungen der Artikulationsstellen sind verdrängt, sie fehlen jetzt ganz. Hingegen werden mimische Gesichtsverzerrungen als Ablenkungen und allerlei Gesten als Spezialhilfsbewegungen eingeführt. Viele scheinen den Wert eines Tics zu haben; sie lassen aber so deutlich den Ursprung der Sprachhilfe erkennen, daß ich die Anwendung dieser Bezeichnung nicht empfehlen möchte. Sie sind gehemmte, wohl abgewogene Gegenwerte für die innerlich vorgestellte Mechanik, den Anfang „schwerer" Laute oder Worte auf einen bestimmt vorgestellten Zeitpunkt zu verlegen. Das Bewegungs- und Kraftübermaß der dynamischen Periode bzw. Methode fehlt diesen Bewegungen durchaus. Unangenehmer und den Gesamteindruck des Sprechenden stärker beeinträchtigend ist die Manier des Mitsprechens mit dem Fragenden, des Ins-WortFallens, desSaugens an denZähnen, des Anzugzerreißens, desTaschentuchzerknüllens, des Rückens auf dem Stuhl und ähnliches mehr. Kinder, bei denen man namentlich in der Schule ein Sich-Einpassen in den „Drill“" wünscht oder gewohnt ist, fallen mit einem Male auf durch Schiefstehen, Buchdrücken, Umsehen, krampfhaftes Lächeln oder rhythmisches Hin- und Herschwanken im Zustande des Antwortensollens, vor allem durch Zeitverlust, und verraten ihre Zugehörigkeit zu dieser Gruppe oft nur schwer.

Die Intensität des ,,inneren Sprechens" ist eine enorme. An Stelle des ",schweren Buchstabens" ist das ,schwere Wort" getreten; das assoziative Gefüge der Begriffe ist erreicht. Die Vorgänge der Rekonstruktion und der assoziativ schwer und schwerer gefrachteten Spraclientschlüsse spielen sich mit fast romanhafter Phantastik und Angst höherer Dimensionen ab. Eine Frage wird nicht einfach angehört; sie wird mit größtmöglicher Geschwindigkeit zu erraten gesucht, und aus zahlreichen Redaktionen der Antwort wird eine "leichte" ausgewählt, wobei noch oder vielmehr schon während ihres Hervorbringens selber noch weitere Redaktionsänderungen vorgenommen werden. Bei spontaner Rede sind Sprechgeschwindigkeit, Denkpausen, Betonung, Ideentiefe und logischer Zusammenhang völlig unnatürlich, d. h. im Vergleich zur Sprache ohne Störungsvorstellung, ebenso im Vergleich zur ursprüng. lichen Gedankenredaktion, die im echten Sinne Antwort, unmittelbar so oder so gemeinte Gegenrede ist. Es fehlen die logischen Obervorstellungen und Leitgedanken, die die menschliche Sprache zu einer dem Äußerungstriebe adäquaten Geistesleistung im Sinne eines Ergebnisses erheben. Die innere Redaktionsänderung der ursprünglichen Gedanken kann so weit gehen, daß glatter Unsinn gesprochen wird, in der Art, daß z. B. die Wiedergabe einer ganz einfachen Lesebuchfabel in einem Mon- 
strum von inhaltsfremder Konfabulation endet, wie ich wiederholt bei Schülern beobachtete. Das fortwährende Ausweichen vor den "schweren" Worten, dieser echt sensorisch-aphatische Vorgang, nimmt von den Begriffen immer mehr logische Schärfe und Bestimmtheit des Urteils weg. Die Überwertigkeit des sprachlichen vor jeder anderen Art von Vorstellen ist auf dem Höhepunkt angelangt. Die Obervorstellungen beim Sprechenden dieser Gruppe sind rein sensorisch-technisch. Diese Periode bzw. diese Aruppe ist die Domäne des sensorischen, d. i. durch Ähnlichkeitsassoziationen im Bestand ähnlicher Empfindungen bedingten Versprechens ${ }^{1}$ ), das die völlige assoziative Auflockerung der inneren Sprache und das Hervortreten aller möglichen sekundären Sprechcharaktere erkennen läßt. Das sogenannte „Poltern“ ist keine Sprachstörung sui generis; der Sprechende ist ein ursprünglich assoziativ Aphatischer, der den extremsten Grad der möglichen sensorischen Sprachrekonstruktion erreicht hat: er entflieht der nur kurz aber stark auftretenden, oft sehr blassen ${ }^{2}$ ) Vorstellung der Störungsmöglichkeit mit einem ganzen Satz, dessen gejagte Aussprache erkennen läßt, daß sich die sensorische Signalstellung eines echten Hilfsmittels bedient, nämlich der Sprechschnelligkeit, eines sog. sekundären Sprechcharakters, und sie willkürlich und übertrieben betätigt. Andere skandieren, indem sie rhythmisch oder silbenmäßig gehackt sprechen.

Einen Übergang von der dynamisch-motorischen zur sensorischaphatischen Gruppe bilden diejenigen Fälle, in denen die Hervorbringung eines starken taktilen Reizes im Sprechapparat zwar die Zugehörigkeit zur dynamisch-motorischen Gruppe einwandfrei erhärtet, in denen aber die Worthervorbringung selber frei von dynamischen Willküren ist. Das sind diejenigen Fälle, in denen fast der ganze Atem ausgepreßt und dann mit dem letzten Rest der Luft unter tunlichster Wortbeschleunigung zu Ende gesprochen wird. Von dieser Methode bis zur sensorischen Signalerwartung ist es nicht mehr weit; diesen Ubergang kann man gelegentlich an ein und demselben Individuum beobachten.

Die Wirkung der beschriebenen Affektlagen auf die Atmung, als diejenige zur Sprachfunktion gehörige Bewegungsautomatie, die ganz allgemein (vgl. die kasuistischen Notizen in meiner zit. Aphasiearbeit S. 512-514) an den Gemütserregungen den unmittelbarsten, unwillkürlichsten und konstitutionell wie funktionell erklärlichsten Anteil hat, ist, auch für den Zustand des Schweigens, ohne weiteres zu verstehen.

1) Vgl. meine Arbeit „Psychologisches über Stottern und Sprechen“, Zeitschr. f. Psychotherap. u. med. Psychol. 3, H. 5, S. $283 \mathrm{ff} .1911$.

2) Das ist kein Widerspruch; der Vorstellungsinhalt kann blaß sein, seine physiologische Wertigkeit wird durch das von ihm abhängige automatisch gewordene Merk - bzw. Bewegungssystem bedingt. Hier ist entweder ein vorgebildeter Automatismus oder sehr starke Verdrängungsarbeit anzunehmen. 
Das Sprechenwollen ist ja in diesen Fällen nur allzu häufig, vielleicht fast regelmäßig das Endergebnis einer mit extremsten Mitteln bewirkten inneren Beherrschung der Affektspannung, einer hochkomplizierten Verdrängungsarbeit und für den Beobachter kaum je ganz durchsichtigen Neueinstellung der ganzen Persönlichkeit zur Situation und zum Sprechakte. Daneben spielt, wie überall im geistigen Geschehen。 die Ausbildung der erwähnten Merksysteme, vom motorischen angefangen bis zu den Registern platter, unangreifbarer, geringe Diskussionsreibungen bietender Allerweltsurteile, eine stets schließlich typenbildende Rolle. Die letzte technische Absicht ist stets die Rekonstruktion einer Sprachleistung, die der inneren Sprache optimal entspricht, sowohl motorisch-dynamisch wie sensorisch-begrifflich. Dabei wird ein Weg durchlaufen, der sich in folgender Art charakterisieren läßt: Aus dem ,schweren“ Buchstaben wird das ,schwere" Wort; vom ,schweren" Wort versteigt sich das sprechende Subjekt zur ,schweren" Sprache, um, nach Erschöpfung aller technischen Möglichkeiten, nach jahrelangen schweren Kämpfen in die Entpersonalisierung einzuwilligen, die das umgestürzte Selbstbewußtsein erzeugt: Der ganze „Ich“-Komplex wird entweder ein „Tabu“, oder, wie man häufig beobachten kann, es kommt zur Prostitution der ganzen sprechenden Persönlichkeit, zur völligen sozialen und moralischen Lähmung, aus der heraus wohl schließlich noch wieder nach den längst abgelegten Masken der dynamisch-motorischen oder der sensorisch - aphatischen Methode gearbeitet wird. Die verdorbene Sprechtat bleibt das Stigma dieser Menschen, deren überhaupt und allgemein letztes „Können“ im vergeblichen Sprechenwollen besteht $\left.{ }^{1}\right)$.

Die dritte Gruppe bzw. die dritte Stufe der assoziativen Aphasie ist, wie vorstehend schon mehrfach angedeutet, der Zustand, in dem der Beschluß zum Spontansprechen affektiv oder assoziativ unter den Einfluß der abstrakt formulierten Störungsvorstellung gerät. Ich habe unter meinen Patienten immerhin einige zwanzig gesehen, die diese Stufe in reiner Form, ohne jede Mischung mit den beiden vorgenannten, zeigten; bei erwachsenen Assoziativ-Aphatischen sind meistens nur Perioden dieses Zustandes vorhanden, oder es handelt sich um Mischung der dynamisch-motorischen und sensorisch-aphatischen Form mit einem dauernd bestehenden schweren psychopatischen Zustande dieser Art. Sprachlich-motorisch ist von diesem assoziativen Betriebe nichts zu bemerken, wenigstens nicht für den Laien; dieser wird die Befallenen meist für ",verrückt" oder verschroben halten aus den bekannten Ursachen innerer Verstimmung ohne Grund, Launenhaftigkeit, Wankelmütigkeit, Charakterschwäche und wie die volkstümlichen Ausdrücke

1) Einen Typ dieser Gruppe habe ich angedeutet in meiner Arbeit in Hans Gross' Archiv S. 172 unten. 
heißen, mit denen man die Psychopathen allgemein benennt. Diejenigen Sekundärataxien, die gelegentlich zur Beobachtung gelangen, scheinen zufällig zu sein; sie sind psychophysisch als blasse motorische Erinnerungen zu bewerten, die in keinem vernünftigen Verhältnis zu der empfundenen Angst und der rasenden Vorstellungsarbeit stehen, die das Gehirn beherbergt. Geste und Mimik sind entweder gesteigert oder unterdrückt, für den geschulten Beobachter aber unverkennbar als nicht dem ursprünglichen Temperament und der natürlichen Gefühlslage entsprechend zu deuten. Wer meine Anamnesen (Aphasiearbeit S. 452 bis 455) liest, wird empfinden, wie sehr es bei Beurteilung dieser Verhältnisse auf die Kenntnis der Vorgeschichte, der ganzen Konstitution, des virtuellen und aktuellen Wissens, des Milieus und der Berufs- und Sonderbeschäftigung ankommt. Man hält sonst vieles für neurotisch, was konstitutionell und psychologisch ist. Die Typen der psychopathischen Veranlagung (imbeziller und debiler Typ, hyperthymischer, depressiver, paranoider und obsessiver Typ) sind, als solche, Ausdrucksformen der Konstitution; ihre Inhalte aber, die von diesen Formen umschlossen werden, weisen die spezifische Zugehörigkeit zu den assoziativaphatischen Zuständen auf. Die genannten Typen sind ja auch durch andere Inhalte alterierbar, modifizierbar und aktivierbar, ohne daß der Typ als solcher ganz verlorenzugehen braucht. Man wundert sich oft, daß man als Spracharzt von Menschen aufgesucht wird, die mit dem besten Willen keine dynamischen Willküren oder sensorisch-aphatische Zustände erkennen lassen, aber bei näherer Analyse zugeben, sie derart stark zu befürchten, daß es in foro doch gelegentlich dazu komme; auch wird oft angegeben, da $\beta$ ein wesentlicher Grad von ,Vorbeidenken“, d. i. von unter assoziativem Einfluß der (abstrakten) Störungsvorstellungen verunstalteter Sprachredaktion, nicht bestehe. Natürlich handelt es sich hier stets um günstige Perioden; nach diesen kommen Zeiten mit starkem Hervortreten der sensorischen oder dynamischen Formen, in denen ungeheure Angst- und Spannungszustände sprachlicher Art sich vorfinden und mit allen möglichen abnormen psychischen Reaktionen vergesellschaften. Die Zugehörigkeit dieser Menschen bzw. einer Periode ihrer Verstimmung zu der genannten Gruppe beweist sich schon durch den Umstand, daß gerade solche davon betroffen sind, die als Geistliche, Lehrer, Telephonisten, Schauspieler u. ä. einen Beruf haben, der sie in die ärgsten Konflikte bringt. Die Spaltung der Persönlichkeit ist nicht nur für den Sprechakt vorhanden, sondern sie umfaßt das ganze Ich. Die Urteilsumwertung betrifft alle Werturteile und hat einen Umfang von phantastischer Größe. Bemerkenswert ist, daß der als Fall 14 Abschn. VI in meiner Arbeit ,Uber die Disposition der Stottererpsyche zu asozialer Entwicklung“" (l. c.) erwähnte Name sich unter den Hauptsowjetkommissaren befindet; auch der Sozialist usw. 
Adler war ein Assoziativ-Aphatischer. Uber die Einzelheiten („Fugues“, Pseudologia phantastica, Zwangshandlungen usw.) habe ich (Aphasiearbeit $u$. a.) ausführlich berichtet. Es handelt sich stets darum, daß der gesamte assoziativ-aphatische Zustand der „Agent provocateur" für diese höheren Stufen ist, für die er aber nicht nur Ursache ist, sondern auch Inhalt und belebendes aktualisierendes Element bleibt. Andere Psychopathen der gleichen Zustandsbilder sind gewissermaßen nur virtuelle Angstneurotiker, deren Konflikte viel seltener sind; umgekehrt hier. Die Abstraktion ,,ich kann nicht sprechen" wirkt, besonders wenn sie im depressiven Affekt einseitig beleuchtet vor der Funktion steht, viel mächtiger als jede entsprechende aus anderen assoziativ-apraktischen Zuständen (z. B. Solistenangst, Platzangst), weil sie schwerer $\mathrm{zu}$ maskieren ist. Aus der technischen ist eine begrifflich-moralische assoziative Aphasie geworden, der auch die weiter unten nachgewiesenen Ausfallserscheinungen anhaften. Um Unklarheiten in der Abgrenzung gegen die rein und unmittelbar psychogenen Aphonien und Aphasien zu beseitigen, sei betont, daß das Charakteristicum der assoziativ-aphatischen Zustände, auch derjenigen dieser letztgenannten Gruppe, die Entwicklung auf der Grundlage der oben geschilderten motorisch-dynamischen Sekundärataxien ist und bleibt; nicht aber handelt es sich um reine Bahnungen, rein affektiv entstandene Psychogenien ohne die Vorstufen niederer assoziativer Beschaffenheit, ohne primär dissoziative Grundlage. Freilich ist bei beiden der fixierende Faktor ein Trieb (hier der Sprachäußerungstrieb, der in jeder Komponente, als Abwehr, als geistiger Formgebungswille, als Klangwohlgefallen usw. getroffen sein kann), aber die Symptome stehen primär nicht in seinem Dienste als auf der Flucht vor extremer Lebensbejahung oder Lebensverneinung gesuchte und willkommene Konfliktslösungen im Sinne Freuds, die also einen quälenden und einen konfliktlösenden Bestandteil von Anbeginn an haben, sondern erst durch und nach Einführen der Sekundärataxien tritt dieser Zustand ein. Das betreffende Individuum ist, wie bei Besprechung der motorisch-dynamischen Form bewiesen wurde, schon vorher sprachlich-dissoziativ gekennzeichnet, die motorisch-dynamischen Willküren sind nicht der primordiale Ausdruck der Sprechangst; sondern die akute Aphonie und die akute Aphasie auf der Grundlage des Schocks sind die Aqquivalente für den letzteren. Die Schreckneurotiker sind aphonisch oder aphasisch aus rein funktioneller, durchaus nicht aber aus assoziativer Ursache; Sekundärhilfen können sie nicht in charakteristischer Weise daraus bilden.

Am häufigsten begegnen wir den abstrakt-psychopathischen Endergebnissen in Verbindung mit den Formen der sensorisch-aphatischen Gruppe; besonders im späteren Lebensalter, dann auch, seltener, verbunden mit den etwaigen konstant gebliebenen Bestandteilen der dyna- 
misch-motorischen Ataxien. Wenn also, um hier noch einmal die falsche Ausdrucksweise anzuwenden, ein „Stotterer", d. i. ein Assoziativ-Aphatischer, beschrieben wird, so muß bei Nennung seiner sprachlichen Symptome jede der drei Gruppen erwähnt werden; alsdann kann sich der fachlich vorgebildete Leser den zu den Symptomen zugehörigen Seelenzustand einigermaßen vorstellen. Die Störungsvorstellung selber hat obsessiven Charakter, sie ist völlig zwangsläufig und durch mächtigen Affektgehalt aus dem kritischen Bewußtsein in die Sphäre der Gläubigkeit gehoben und zum Dogma erhöht, das jegliche ethische, moralische und naturwissenschaftliche Werte hemmungs- und schamlos umwertet ${ }^{1}$ ). Der begleitende Affektzustand, der sich zu Anfang auf Buchstaben und Wort bezog (,Verbalaffekt"), hat die Begriffe einbezogen und die Komplexbildung besonders bei der sensorisch-aphatischen Gruppe bewirkt; jetzt hat er sich auf die Allgemeingefühle ausgedehnt. Wenn die psychopathische Konstitution als solche an und für sich eine Übererregbarkeit vieler höheren Gefühle bedingt, so daß pathologische Affektstärken zu abnormen sozialen und ethisch-moralischen Einstellungen führen können, so ist, wie die Beobachtung und dịe Erfahrung lehrt, dieStärke und Färbung der aus den sprachlich-dissoziierten Komplexen stammenden Affektmasse keineswegs immer auf den gleichen Gefühlston eingestimmt. Die Eigenart der assoziativen Aphasie bleibt also auch hierin gewahrt gegenüber allgemein psychopathisch bedingten Gleichgewichtsstörungen. Durchaus nicht alle, nicht einmal (schätzungsweise) die Mehrzahl der Assoziativ-Aphatischen stellen vermöge ihres psychischen Funktionalismus ihre Symptome so tendenziös in den Vordergrund ihrer sozialen Erscheinung, wie dies bei den Schreck- und Angstneurotikern der Fall ist. Der Assoziativ-Aphatische hat primär keine Krankheitsmotive; die bekommt er erst im ausgebildeten $\mathrm{Zu}$ stande, ausgenommen die durch Nachahmung dis- und späterhin assoziativ Gewordenen, bei denen der Trieb zum Anders- und Besonderssein primär vorhanden war. Das sei bestimmt ausgesprochen; denn bei diesen ist eine Abwehrstimmung, ein Schutzinstinkt das die Nachahmung fixierende Agens. Der aus der Lehre der Psychoanalytiker stammende Satz, daß das nervöse Symptom ein Versuch zur Konfliktlösung sei (Antrieb: Selbsterhaltungs- bzw. Betätigungstrieb, Angriffstrieb, Sprachäußerungstrieb; Hemmung: Ereignis, Erinnerungsbild, das einen irgendwie beschaffenen Unwert den eben genannten Trieben in den Weg schiebt; Konflikt: Differenz- bzw. Interferenzerscheinung aus dem Wunsch, beides gleichzeig zu besitzen, je nach Antrieb aus der einen oder der anderen Gruppe), hat hiernach auch für die Assoziativ-Aphatischen erst im höheren Stadium Gültigkeit, keineswegs aber für den primär-disso-

1) Als Unikum sei ein Referendar erwähnt, der fast alle ethischen und moralischen Werte umgewertet hatte und imbezill war. 
ziativen Anfang und die für alles weitere so grundsätzlich bestimmende dynamisch-motorische Stufe.

Daß die dauernde Affektivität eine ganze Reihe von psychischen, auch moralischen und sexuellen Abnormitäten, auch frühzeitig schon erzeugen kann, sei nebenbei bemerkt; es erübrigt sich nach dem vorausgehend Gesagten darauf einzugehen, da trotz aller gelegentlich aufdeckbaren Zusammenhänge die Ursächlichkeitsverhältnisse sich grundsätzlich nicht anders verhalten als hier angegeben. Die Häufigkeit war in meiner Klientel gering; viele, besonders erotische, Psychotraumen sind bei weitem später als die primären Sprachdissoziationen; anderes wird während der Psychoanalyse vermöge Gefühlsübertragung nachträglich bewertet. Man ist hier sehr leicht geneigt, ex juvantibus zu schließen.

Manche sehr temperamentvolle Assoziativ-Aphatiker der höherer Lebensalter $(30-40)$ scheinen, bewußt und unbewußt, sich gern zu ermüden. Sie finden, daß nach durchschwärmter Nacht, nach reichlichem Alkoholgenuß (nicht im Rausch) die Affektlage günstiger, das Auftreten der Störungsvorstellungen seltener und blasser ist. Es kann sich hierbei um einen sowohl konstitutionellen wie auch reflexiv bedingten Mechanismus handeln, der vom „Betriebsgefühl“", vom Gefühl für optimalen Besitz der geistigen Willenserzeugnisse abhängig sein mag; nicht gemeint ist die Abhängigkeit der Störungsvorstellung vom Zustand der Müdigkeit, des Rausches usw.; da pflegt es umgekehrt zu sein.

\section{VI.}

Die Ausfallserscheinungen der Assoziativ-Aphatiker sind meines Wissens noch nie angegeben worden. Theoretisch betrachtet widerspricht es der Vorstellung von der Einstellung des aktuellen Wissens, $\mathrm{da} ß$ die hier geschilderten Verhältnisse mit einer Normalwertigkeit der geistigen Alltagsleistung und Anpassungsfähigkeit verbunden sein könnten. Die unausgesetzt motivierte sprachliche Prosexie ist als Ursache anzugeben, die Entwicklung zum Zustand geschieht durch Fortbestehen der Ursachen.

Schon in der Kindheit, und bei den ersten Anfängen überhaupt, fällt die Verringerung des Kausalitätsbedürfnisses ins Auge. Dem psychischen Gesamtfunktionalismus wird, durch Affektverlagerung und Erschöpfung in Vergeblichkeiten, das Streben und der Trieb nach Kenntnis der alltäglichsten Zusammenhänge entzogen. Je stärker dies stattfindet, je größere Geneigtheit die psychopathische Konstitution hierfür besitzt, um so auffallender bildet sich ein Zustand von Leichtbis Dummgläubigkeit aus, der sich oft wie echte Imbezillität oder Debilität ausnimmt. Auch häufige und folgenschwere Negativismen kommen zur Beobachtung. 
Das kindliche Haften am sprachlich Sinnlichsten, an den Sprechbewegungen hat häufiges Versagen der äußeren Aufmerksamkeit zur Folge. Besonders in der Schule tritt dies in Erscheinung. Die Verläßlichkeit des Gedächtnisses und die Merkfähigkeit sind herabgesetzt, die Motivierung der Aufmerksamkeit ist oft überdeckt von den Affekten des Störungskomplexes, die Folge ist sprunghafte und ungleichmäßige Auffassung, oft Begabungen und das Gegenteil vortäuschend.

Die überaus große Schwäche der Auffassung realer Werte, der Bewertung von Erfahrungen haben ihren Grund in einem echt sprachlichen Symptom dieses Zustandes, in der schon erwähnten (apperzeptiven) Hypersymbolie. Da die Worte ähnlichdeutig und mehrdeutig verwendet werden, da infolge des schon frühzeitig verringerten Kausalitätsbedürfnisses die Begriffsbildung unscharf ist, so sind auch verminderte Hemmungen aus dem Gebiete der logischen Gefühle vorhanden (AssoziativAphatiker sind z. B. selten gute Mathematiker), und daher wird weder einfach gelesen noch zugehört, sondern stets nur erraten, und die Welt der schmerzhaften Ursachen und Wirkungen wird ebenso umgangen und nur mit Fingerspitzen angefaßt wie ein ,schwerer" Buchstabe. Man kann bei fortschreitender Auflösung der Störungsvorstellung kurvenmäßig nachweisen, wie die Zahl des Versprechens beim Vorlesen und Spontansprechen bis zur Norm absinkt. Ich empfehle diese Beobachtung sehr; denn sie macht mehr wie andere Untersuchungen, bei denen besondere Einstellungen der Aufmerksamkeit ungünstig auf den Vorstellungstyp usw. einwirken, die Verhältnisse durchsichtig. Denhardt ließ ein geeignet geschnittenes Papierstückchen so über dic dem Blick abzusperrenden Zeilen des Buches schieben, daß das Vorauseilen der Perzeptionssucht unmöglich wurde; da hierdurch gleichzeitig eine enorme Verminderung der für die Reflexion sonst verbrauchten Zeit herbeigeführt wird, so dient schon dies unscheinbare, aber geniale Mittel zur wirksamsten Unterstützung der übrigen Heilmaßnahmen. Während des phonetisch-zusammenhängenden Weitersprechens wird dem psychischen Zusammenhang auf diese Weise die Zeit zum Einschieben der Störungsvorstellung einfach mechanisch entzogen und so der Hypersymbolie gleichzeitig entgegengearbeitet. Es bedarf der ganzen Geschicklichkeit des Spracharztes, in den U'bungsstunden eine entsprechende Atmosphäre von Auffassungsstrenge auch für die freie Rede aufzurichten und aufrechtzuerhalten, da namentlich die Erwachsenen, die ohne ein „Parterre" von Leuten, denen ihre Abnormitäten imponieren, zunächst nicht leben können, außerordentlich hartnäckig in der Verfolgung ihrer Denkgewohnheiten sind und danach streben, ,,ihren" Fehler zu demonstrieren, infolgedessen auf ein „,bißchen“ Versprechen keinerlei Wert legen,

Ich habe a. a. O. darüber berichtet, daß die Art des Versprechens ungemein charakteristisch für die ,,begriffliche Entwertung des Wortes“", 
für seine psychologische Entwertung zum Bewegungsvorgang ist. Es treten nämlich alle möglichen Empfindungs- und Buchstabenassoziationen dabei hervor, die weit mehr wie sinnentstellend sind und direkt den „roten Faden“ der sich von einer gleichartigen Störung bzw. „Schwierigkeit“" zur anderen weiterziehenden Störungsvorstellung verkörpern.

Aus dieser Schwäche der Auffassung entwickelt sich später eine allgemeine und besonders die eigene Sprachleistung betreffende Urteilsschwäche und Eitelkeit, die beide ihren Grund in einem teils subjektiv, teils objektiv begründeten Egozentrismus haben. Alle komplex gewordenen Psychopathen sind an und für sich stark auto- und allosuggestibel; so auch hier. Sie haben wenige und unscharfe soziale Begriffe, da sie zum täglichen Leben oft nur mit Teilvorstellungen in Beziehung stehen. Das Nebelhafte, Unklare und Wunderbare, das die Logik und die Kritik, ja selbst die Erfahrung wenig bestimmt herausfordert und einen beträchtlichen Bezirk dieser ,"Terra incognita" für die Entdeckung des längst Bekannten übrigläßt, ist ihr Element. Sie sind eigensinnig und doch, trotz schwerer Lenkbarkeit für reale Anforderungen, lenksam für diejenigen, die sich ihrer Gläubigkeit zu bemächtigen verstehen und Unverstandenes zum Dogma zu erheben vermögen. Sie sind und bleiben die Kerntruppe derer vom „Esprit d' à peu-près“.

Die Assoziativ-Aphatiker teilen, infolge der dauernden Gereiztheit ihrer Werturteile durch gesteigerte Affektlage und infolge der großen Angreifbarkeit ihrer ganzen Sprach- und Lebenseinstellung, mit den anderen gleichgewichtgestörten Psychopathen auch die große seelische Verwundbarkeit infolge des Mangels an wiederherstellenden Gefühls- und Vorstellungsautomatien.

Von der Angst ist schon gesprochen worden. Sie ist die Interferenzerscheinung zwischen den gegensätzlichen Trieben, die einerseits die Sprachäußerung, andererseits die Hemmung vor den dynamischen Willküren antreiben; Unlust und Verstimmung wurzeln aber hier durchaus nicht immer nur in den Vorstellungskomplexen des Störungsbewußtseins (s. o.), sondern sehr häufig, besonders bei den Erwachsenen, tief in der gesamten seelischen Konstitution. Periodische Verstimmungen weisen oft auf Cycliker hin, auch manische Perioden mit positivem Gefühlston werden beobachtet, die vom psychiatrischen Standpunkt ebensowohl als abnorm bezeichnet werden müssen wie die depressiven, obwohl in den hyperthymischen Perioden meist viel besser gesprochen, aber desto abnormer gelebt und produziert wird. Hier geht der Rahmen der Psychopathie über das Bild des assoziativ-aphatischen Zustandes hinaus; in den meisten Fällen allerdings ist die psychopathische Veranlagung völlig ausgebaut vom aphatischen Zustande.

Als direkte Symptome der inneren Sprache ergeben sich somit: 
1. Grammatikalische und logische Schwäche.

2. Pseudologia phantastica der Assoziativ-Aphatischen.

3. Verringerung des Wortbestandes, Erschwerung der Wortfindung.

4. Begriffsschwäche.

5. Hypersymbolie (Assoziierung der Wortklänge mit Teilvorstellungen).

6. Verschiefung der Werturteile (z. T. durch Unlustgefühlsübertragung auf den virtuellen Wortschatz).

Die Assoziativ-Aphatiker sind sämtlich ausgezeichnet durch ein gestörtes Selbstbewußtsein. Funktionsunsicherheit, Umwertung des Sprachwertes, Gedächtnisschwäche und Mangel an Wiederherstellungsfähigkeiten gegenüber der gestörten Funktion sind die Bestandteile. Zustand und Betrieb sind geschwächt, gestört und nach der krankhaften Seite gesteigert. Die Spaltung der Persönlichkeit findet, wie schon angedeutet, ihre Endform in der völligen sprachlichen und sozialen Preisgabe der Existenz. Das „Leben" wird mit allen Symptomen der Neurose bekämpft. Der Wille zur Krankheit ist eine alltägliche Erscheinung und findet seinen Grund in der Störung des Ichbewußtseins. Der Beruf wird als Maske getragen, aber er verwächst nicht mit der geistigen Physiognomie des Menschen, denn diese ist proteusartig wandelbar. Der Wunsch zur Heilung fehlt, weil dies Wort nur abstrakt, d. i. als Fertiges, geschaut werden kann und deshalb, wie immer in ähnlichen Fällen, ein wegloses Neuland bleibt, das mit keinem der bekannten Begriffe angegangen werden kann. Die Gesundheit bleibt ein phantastischer Traum, der von der ,feindlichen“, d. i. der gesunden Hälfte der Psyche geträumt wird. Auch bei dem Versuch, das Bild der Normalität dem Leidenden nahezubringen, stößt man auf starke Abwehren, die aus der konstitutionellen Abgestimmtheit der Persönlichkeit stammen können. Die Störungsvorstellung erweitert sich zum Störungskomplex, dieser zum Störungsbewußtsein; eines schwarzen Tages übernimmt das Störungsbewußtsein Namen, Titel und Rechte des Ich, während das ursprüngliche, auf Normalfunktion aufgebaute Ich nichts weiter als die schmerzliche Erinnerung an müheloses Normalsprechen für sich hat, von dem es nach Sachlage unter keinen erheblichen Umständen Gebrauch machen kann. Alle übrigen höheren Seelenfunktionen sind unausgebildet geblieben, und so war kein wesentliches Hindernis vorhanden, das dem Umsturze wehren konnte. Das ethische und soziale Anderssein, das durch Sprachanomalie hervorgebracht wird, berührt sich mit demjenigen aus der psychopathischen Anlage; diese selbst ist aber meist ohne selbständige Züge, da die Sprachkomplexe alles aktuelle Leben erfüllen. Man findet, wie gesagt, beide Zustandsbilder: assoziativ-aphatischer Zustand, plus Psychopathie mit selbständigen Zügen, und: assoziativ-aphatischer Zustand mit den Inhalten 
des Störungsbewußtseins der Aphasie und den Reaktionen der Psychopathie, ohne nebenhergehende selbständige Züge des betreffenden psychopathischen Typs; also in diesem Falle: dyslogische Form der Angstpsychose.

\section{VII.}

Die Kenntnis dieser Verhältnisse gehört nicht in das Gebiet des Hals-, Nasen- und Ohrenarztes. Sie ist ein selbständiges Teilgebiet der Psychiatrie, nämlich der Medizinischen Psychologie, und der ärztlichen Pädagogik hinsichtlich ihrer Behandlung. Die psychiatrische oder die medizinische Klinik haben, besonders nach dem Kriege, Ursache, dieses Gebiet zu besitzen und die dahingehörigen Fälle zu sammeln. Die soziale Gefährdung dieser Leidenden ist namentlich in jetzigen Zeiten der sozialen Unbeständigkeit und der Entwertung der Gesellschaftsinteressen so groß, daß diese Gefahr auf die Sozialität zurückschlagen kann, wenn nicht stark zugegriffen und das Gebiet endlich von Kurpfuschern und Unsachverständigen gereinigt wird. Zahlreiche Fortbildungseinrichtungen entbehren der geeigneten Lehre; zahlreiche Hilfseinrichtungen arbeiten mit ungenügend und ungeeignet ausgebildeten Kräften. Die Pathopsychologie wird sich an der Klinik und an den Fürsorgeeinrichtungen ihr Feld zu erobern haben. 